\title{
Laplace type operators: Dirichlet problem
}

\author{
WOJCIECH KOZŁOWSKI
}

\begin{abstract}
We investigate Laplace type operators in the Euclidean space. We give a purely algebraic proof of the theorem on existence and uniqueness (in the space of polynomial forms) of the Dirichlet boundary problem for a Laplace type operator and give a method of determining the exact solution to that problem. Moreover, we give a decomposition of the kernel of a Laplace type operator into $\mathrm{SO}(n)$-irreducible subspaces.
\end{abstract}

Mathematics Subject Classification (2000): 35J25 (primary); 34K10, 35J67 (secondary).

\section{Introduction}

In geometric and analytic investigations there appears, in a natural way, a whole class of elliptic self-adjoint operators of form

$$
L=L_{a, b}=a d \delta+b \delta d, \quad a, b>0,
$$

called Laplace type operators. If $a=b=1$ then $L=\Delta=d \delta+\delta d$ is simply the Laplace-Beltrami operator. Another example is the Laplace-Ahlfors operator $S^{\star} S$. Originally, the Laplace-Ahlfors operator was defined as an operator acting on the space of smooth vector fields in $\mathbb{R}^{n}: S^{\star} S=\frac{1}{2} \Delta+\frac{n-2}{2 n} \operatorname{grad}$ div. If $n=3, S^{\star} S=0$ reduces to the elasticity equation considered by H. Weyl ([14]). (For more details we refer to [1] or [11]). Because of the natural duality between the space of vector fields and 1 -forms in $\mathbb{R}^{n}$, the operator $S^{\star} S$ can be identified with the operator $L=L_{\frac{n-1}{n}, \frac{1}{2}}=\frac{n-1}{n} d \delta+\frac{1}{2} \delta d$, acting on the space of 1-forms in $\mathbb{R}^{n}$. A generalization of the Ahlfors-Laplace operator to Riemannian manifolds was done by A. Pierzchalski (see $[9,10])$. It is worth to note that if a Riemannian manifold is Ricci flat then the Ahlfors-Laplace operator has exactly the form $L_{\frac{n-1}{n}, \frac{1}{2}}$.

Laplace type operators have some properties similar to $\Delta$. For example $L_{a, b}=$ $(\sqrt{a} \delta+\sqrt{b} d)^{\star}(\sqrt{a} \delta+\sqrt{b} d)$, where the star denotes the operator adjoint with Received July 20, 2006; accepted in revised form December 12, 2006. 
respect to the inner product given by an integral. Despite similarities, some of the important features of $L$ may be quite different than those of $\Delta$. The reason lies in a shape of the leading symbol of $L$. By contrast to $\Delta, L$ is not of metric symbol, if $a \neq b$.

In this paper we investigate Laplace type operators in the Euclidian space $\mathbb{R}^{n}$. Since partial derivation of a polynomial is an algebraic operation, both the differential $d$ and co-differential $\delta$ in the space $\Lambda^{p}$ of polynomial $p$-forms in $\mathbb{R}^{n}$ can be defined in a pure algebraic way. In particular, any Laplace type operator $L=L_{a, b}=a d \delta+b \delta d$ may be defined in this manner. There appears a natural question, whether one can investigate the operator $L$ using pure algebraic methods. In particular, whether one can obtain the theorem on existence and uniqueness purely algebraically for such operators. In this paper we give an affirmative answer.

The main results are: Theorem 4.5 on existence and uniqueness of solutions to the Dirichlet boundary problem for $L$ and the unit sphere in $\mathbb{R}^{n}$, and Section 4.4 where we give an algorithm for solving Dirichlet boundary problem explicitly. The algorithm involves pure algebraic operations only.

In the whole paper (except Section 5 which is a complement to theory developed in Section 2.1-3.3) we do not use any analysis, at all. Surprisingly enough, to the best of the author's knowledge, no book on differential equations or spherical harmonic, contains an algebraic proof on existence and uniqueness of solutions to the Dirichlet boundary problem, even in the simplest case $\Delta \varphi=0,\left.\varphi\right|_{S}=f$, where $f$ is a polynomial 0 -form, i.e., polynomial in $\mathbb{R}^{n}$. The most algebraic proof we found is contained in the Krylov's book [7], nevertheless the author applies the Maximum Principle which is proved analytically.

The main tool we construct is the decomposition (3.16) of the $\mathfrak{L}_{k}^{p}=\operatorname{ker}(L$ : $\Lambda_{k}^{p} \rightarrow \Lambda_{k}^{p}$ ) into four mutually orthogonal (with respect to some special inner product (2.3)) subspaces. This decomposition seems to be of independent interest. Here $\Lambda_{k}^{p}$ denotes the space of polynomial $p$-forms that coefficients are homogeneous of degree $k$. The important tool in getting the decomposition is Theorem 2.7 which asserts that $d^{\star}=\iota_{v}$ and $\delta^{\star}=-\varepsilon_{v}$. This fact was observed by Antoni Pierzchalski.

In the special case $L=S^{\star} S$ and $p=1$ the decomposition (3.16) reduces to the decomposition of ker $S^{\star} S$ obtained by H. M. Reimann in [11]. It was later used by A. Lipowski in [8], where the author considered Neumann type boundary conditions for $S^{\star} S$.

In Section 4.3 the projections formulae onto the four subspaces from (3.16) are given. Applying this formulae together with the method from the proof of theorem on existence solution to the Dirichlet boundary problem we build an algorithm of solving Dirichlet problem $L \varphi=0,\left.\varphi\right|_{S}=\left.\omega\right|_{S}$, explicitly. Moreover, we give an example (see Example 4.12) of direct application of the method. This algorithm could be applied for solving the Dirichlet problem by computers.

In Section 5 we investigate the decomposition of $\mathfrak{L}_{k}^{p}$ from the representation theory point of view. It turns out that (3.16) may be reducible in general. The main result of this section - Theorem 5.5 - contains the decomposition $\mathfrak{L}_{k}^{p}$ into $\mathrm{SO}(n)$ irreducible subspaces. In the special case $p=1$ and $L=\Delta$ the decomposition 
from Theorem 5.5 reduces to those from [11] and [6]. In Section 5 we use algebraic tools constructed in Section 2.1-3.3 only with the one exception, namely, we apply Theorem A from [4].

This paper was inspired by the articles [11] of H. M. Reimann and [4] of G. B. Folland.

Acknowledgements. The author would like to thank very much to Professor Antoni Pierzchalski for helpful discussions.

\section{Preliminaries}

\subsection{Polynomial forms}

Suppose $n$ is non-negative integer and $n \geq 2$. If $x=\left(x^{1}, \ldots, x^{n}\right) \in \mathbb{R}^{n}$ and $\alpha=$ $\left(\alpha_{1}, \ldots, \alpha_{n}\right)$ is a multi-index then $x^{\alpha}=\left(x^{1}\right)^{\alpha_{1}} \cdots\left(x^{n}\right)^{\alpha_{n}}$ and $|\alpha|=\alpha_{1}+\cdots+\alpha_{n}$. Put $\partial_{i}=\frac{\partial}{\partial x^{i}}, \partial_{i, j}^{2}=\partial_{i} \circ \partial_{j}$ and $D^{\alpha}=\left(\partial_{1}\right)^{\alpha_{1}} \circ \cdots \circ\left(\partial_{n}\right)^{\alpha_{n}}$.

Recall first the basic properties of homogeneous polynomials. For more details we refer to $[3,12,2]$. Let $\mathcal{P}_{k}$ denote the space of all (real-valued) homogeneous polynomials in $\mathbb{R}^{n}$ of degree $k$. As a consequence of homogeneity we obtain, so called, Euler property: $k f(x)=\sum_{i=1}^{n} x^{i} \partial_{i} f(x)$. If $f \in \mathcal{P}_{k}$ has a form $f(x)=$ $\sum_{|\alpha|=k} a_{\alpha} x^{\alpha}$ we define differential operator $f(D)=\sum_{|\alpha|=k} a_{\alpha} D^{\alpha}$. Obviously, $f(D): \mathcal{P}_{l} \rightarrow \mathcal{P}_{l-k}$.

Define the inner product $(\cdot, \cdot)=(\cdot, \cdot)_{k}$ in $\mathcal{P}_{k}$ as follows; $(f, g)=f(D) g$, for $f, g \in \mathcal{P}_{k}$. Clearly, for any $f \in \mathcal{P}_{k}, g \in \mathcal{P}_{l}$ and $h \in \mathcal{P}_{k+l},(g f, h)_{k+l}=$ $(f, g(D) h)_{k}$. In particular, $\left(x^{j} f, h\right)=\left(f, \partial_{j} h\right)$. It means that multiplication by $g$ and operator $g(D)$ are formally adjoint each to the other.

For any $x \in \mathbb{R}^{n}$ let $|x|$ denote Euclidean norm in $\mathbb{R}^{n}$. The polynomial $r^{2}$ defined by $r^{2}(x)=|x|^{2}$ is a member of $\mathcal{P}_{2}$. Differential operator $\Delta=-r^{2}(D)$ is nothing but the classical Laplace operator. Let $\mathcal{H}_{k}=\left\{h \in \mathcal{P}_{k}: \Delta h=0\right\}$ be the space of all harmonic homogeneous polynomials of degree $k$. Clearly, $\mathcal{H}_{k}=\{h \in$ $\left.\mathcal{P}_{k}: r^{2}(D) h=0\right\}$. By Euler property it follows easily that for any $f \in \mathcal{P}_{k}$,

$$
\Delta\left(r^{2} f\right)=-2(n+2 k) f+r^{2} \Delta f
$$

Since multiplication by $-r^{2}$ and $\Delta$ are formally adjoint, we obtain

$$
\mathcal{P}_{k}=\mathcal{H}_{k} \oplus^{\perp} r^{2} \mathcal{P}_{k-2}
$$

where $\oplus^{\perp}$ denotes orthogonal direct sum. In particular, $\operatorname{dim} \mathcal{H}_{k}=\operatorname{dim} \mathcal{P}_{k}-$ $\operatorname{dim} \mathcal{P}_{k-2}$. Thus $\operatorname{dimim}\left(\left.\Delta\right|_{\mathcal{P}_{k}}\right)=\operatorname{dim} \mathcal{P}_{k}-\operatorname{dim} \mathcal{H}_{k}=\operatorname{dim} \mathcal{P}_{k-2}$ 
As a direct consequence of the formula (2.2) we obtain:

Proposition 2.1. For each $f \in \mathcal{P}_{k}$ there exist unique polynomials $h_{j} \in \mathcal{H}_{k-2 j}$, $0 \leq j \leq m_{k}=[k / 2]$ such that $f=h_{0}+r^{2} h_{1}+\cdots+\left(r^{2}\right)^{m_{k}} h_{m_{k}}$.

Consider any $p$-form $\omega$ in $\mathbb{R}^{n}, p \geq 0$. If $p=0$ we identify $\omega$ with a function on $\mathbb{R}^{n}$. Assume that any $p$-form, $p<0$, is the zero form. If $p \geq 1$ then $\omega$ has unique expression

$$
\omega=\frac{1}{p !} \sum_{i_{1}, \ldots, i_{p}=1}^{n} \omega_{i_{1}, \ldots, i_{p}} d x^{i_{1}} \wedge \cdots \wedge d x^{i_{p}}
$$

where the functions $\omega_{i_{1}, \ldots, i_{p}}=\omega\left(\partial_{i_{1}}, \ldots, \partial_{i_{p}}\right)$, called coefficients, are skew-symmetric with respect to indices. A $p$-form $\omega$ is called polynomial $p$-form if $\omega_{i_{1}, \ldots, i_{p}}$ 's are polynomials. Denote by $\Lambda^{p}$ vector space of all polynomial $p$-forms in $\mathbb{R}^{n}$. Although, in this paper, we consider only polynomial forms, some properties, formulae, theorems etc. hold for forms with differentiable (or even arbitrary) coefficients. For this reason designation "polynomial form" will be used only if a property, formula, theorem etc. holds only for forms with polynomial coefficients.

If $\omega$ and $\eta$ are any $p$-forms defined on a subset $Z \subset \mathbb{R}^{n}$ then $\omega \eta$ will denote point-wise inner product of that forms, i.e., $\omega \eta$ is a function $Z \rightarrow \mathbb{R}$ defined by

$$
\omega \eta=\frac{1}{p !} \sum_{i_{1}, \ldots, i_{p}=1}^{n} \omega_{i_{1}, \ldots, i_{p}} \eta_{i_{1}, \ldots, i_{p}} .
$$

A polynomial $p$-form $\omega$ is called homogeneous if all coefficients are from $\mathcal{P}_{k}$, for some $k$. Such a form will be called $(p / k)$-form. Denote by $\Lambda_{k}^{p}$ the vector space of all ( $p / k)$-forms. Clearly, $\Lambda_{k}^{p}$ is a finite dimensional vector space and $\operatorname{dim} \Lambda_{k}^{p}=$ $\left(\begin{array}{l}n \\ p\end{array}\right) \operatorname{dim} \mathcal{P}^{k}$. Manifestly, $\Lambda_{k}^{0}=\mathcal{P}^{k}$, and $\Lambda_{k}^{n}$ is, in a natural way, isomorphic to $\mathcal{P}^{k}$. Moreover, it is convenient to put $\Lambda_{k}^{p}=\{0\}$ if either $p<0$ or $k<0$.

Equip the space $\Lambda_{k}^{p}$ with the inner product $(\cdot \mid \cdot)=(\cdot \mid \cdot)_{p, k}$ as follows; for any $(p / k)$-forms $\omega$ and $\eta$ we put

$$
(\omega \mid \eta)_{p, k}=\frac{1}{p !} \sum_{i_{1}, \ldots, i_{p}=1}^{n}\left(\omega_{i_{1}, \ldots, i_{p}}, \eta_{i_{1}, \ldots, i_{p}}\right)_{k},
$$

where $\omega_{i_{1}, \ldots, i_{p}}$ 's and $\eta_{i_{1}, \ldots, i_{p}}$ 's denote coefficients of $\omega$ and $\eta$, respectively. Notice that $(\cdot \mid \cdot)_{0, k}$ and $(\cdot, \cdot)_{k}$ coincide.

Consider the vector field $v_{x}=x^{1} \partial_{1}+\cdots+x^{n} \partial_{n}$ and the $(1 / 1)$-form $v_{x}^{\star}=$ $x^{1} d x^{1}+\cdots+x^{n} d x^{n}$. One sees that $v^{\star} v=r^{2}$. Let $\varepsilon_{v}$ denote exterior multiplication by $\nu^{\star}$, i.e., $\varepsilon_{\nu} \omega=\nu^{\star} \wedge \omega$. Its adjoint with respect to the pointwise inner product is denoted by $\iota_{\nu}$, i.e., $\left(\varepsilon_{\nu} \omega\right) \eta=\omega\left(\iota_{\nu} \eta\right)$. If $\omega$ is a $p$-form then $\iota_{\nu} \omega$ is $(p-1)$-form defined by $\iota_{\nu} \omega=\omega(\nu, \cdot, \ldots, \cdot)$ if $p \geq 1$ and $\iota_{\nu} \omega=0$, if $p=0$, and $\varepsilon_{\nu} \omega=v^{\star} \wedge \omega$. 
It is clear that for any $(p / k)$-form $\omega, \iota_{\nu} \omega \in \Lambda_{k+1}^{p-1}$ and $\varepsilon_{\nu} \omega \in \Lambda_{k+1}^{p+1}$. Manifestly, $\iota_{v}^{2}=\iota_{v} \circ \iota_{v}=0$ and $\varepsilon_{v}^{2}=\varepsilon_{v} \circ \varepsilon_{v}=0$. Moreover, ([5, page 63]) $\iota_{v}$ is graded derivation of degree -1 . As an easy consequence of the definitions we obtain that for any $p$-form $\omega$,

$$
\begin{aligned}
\left(\varepsilon_{\nu} \omega\right)_{i_{0}, \ldots, i_{p}} & =x^{i_{0}} \omega_{i_{1}, i_{2}, \ldots, i_{p}}-x^{i_{1}} \omega_{i_{0}, i_{2}, \ldots, i_{p}}-\cdots-x^{i_{p}} \omega_{i_{1}, i_{2}, \ldots, i_{0}}, \\
\left(\iota_{\nu} \omega\right)_{i_{2}, \ldots, i_{p}} & =\sum_{i=1}^{n} x^{i} \omega_{i, i_{2}, \ldots, i_{p}} .
\end{aligned}
$$

Let $\star$ denote Hodge star operator. Recall that for any $p$-form $\eta, \star \eta$ is the unique $(n-p)$-form such that for any $p$-form $\omega$,

$$
\omega \wedge \star \eta=(\omega \eta) d x^{1} \wedge \cdots \wedge d x^{n} .
$$

This operator is an isometry, i.e, $(\star \omega)(\star \eta)=\omega \eta$ and satisfies the identity $\star^{2}=$ $\star \star=(-1)^{p(n-p)}$ on the space of $p$-forms.

Let $x_{0} \in \mathbb{R}^{n}$. We say that $\omega$ is tangential (respectively normal) at $x_{0}$ if $\left(\iota_{\nu} \omega\right)_{x_{0}}=0$ (respectively $\left.\left(\varepsilon_{\nu} \omega\right)_{x_{0}}=0\right)$. Clearly, each form is tangential and normal at $0 \in \mathbb{R}^{n}$. Take now any subset $Z \subset \mathbb{R}^{n}$. We say that $\omega$ is tangential (respectively normal) on $Z$ if $\omega$ is tangential (respectively normal) at each point $z \in Z$.

Take any $p$-form $\omega$. Since $\iota_{v}$ is graded derivation of degree -1 and $\nu^{\star} v=r^{2}$, we have $\iota_{\nu} \varepsilon_{\nu} \omega=r^{2} \omega-\varepsilon_{\nu} \iota_{\nu} \omega$. Hence we obtain

$$
\left(\iota_{\nu} \varepsilon_{v}+\varepsilon_{\nu} \iota_{v}\right) \omega=r^{2} \omega .
$$

It follows that each $\omega$ may be uniquely written as

$$
\omega=\omega^{\mathrm{T}}+\omega^{\mathrm{N}}
$$

where $\omega^{\mathrm{T}}$ are $\omega^{\mathrm{N}}$ tangential and normal, respectively. Outside $0 \in \mathbb{R}^{n}$, we have $\omega^{\mathrm{T}}=\left(1 / r^{2}\right) \iota_{\nu} \varepsilon_{\nu} \omega$ and $\omega^{\mathrm{N}}=\left(1 / r^{2}\right) \varepsilon_{\nu} \iota_{\nu} \omega$.

Corollary 2.2. Let $\omega$ be a p-form. Take any $x_{0} \neq 0, x_{0} \in \mathbb{R}^{n}$. If $\omega$ is both tangential and normal at $x_{0}$, then $\omega_{x_{0}}=0$. In particular, if the polynomial form $\omega$ is both tangential and normal on $\mathbb{R}^{n}$ then $\omega=0$.

Proof. We have $\left(\iota_{\nu} \omega\right)_{x_{0}}=\left(\varepsilon_{\nu} \omega\right)_{x_{0}}=0$. Thus $\left(\varepsilon_{\nu} \iota_{\nu} \omega\right)_{x_{0}}=\left(\iota_{\nu} \varepsilon_{v} \omega\right)_{x_{0}}=0$. Hence the formula (2.4) implies that $\left(r^{2} \omega\right)_{x_{0}}=\left|x_{0}\right|^{2} \omega_{x_{0}}=0$. Since $x_{0} \neq 0, \omega_{x_{0}}=0$.

For any $a, b \in \mathbb{R}$ define the linear operator $\mathfrak{l}_{a, b}$ by $\mathfrak{l}_{a, b}=a \varepsilon_{v} \iota_{v}+b \iota_{v} \varepsilon_{v}$. Clearly, $\mathfrak{l}_{a, b}: \Lambda^{p} \rightarrow \Lambda^{p}$ and $\mathfrak{l}_{a, b}: \Lambda_{k}^{p} \rightarrow \Lambda_{k+2}^{p}$.

Proposition 2.3. If $a, b \neq 0$ then $\mathfrak{l}_{a, b}: \Lambda^{p} \rightarrow \Lambda^{p}$ is one-to-one.

Proof. Take any $p$-form $\omega$ and suppose that $\mathfrak{l}_{a, b} \omega=0$, i.e., $a \varepsilon_{v} \iota_{\nu} \omega=-b \iota_{\nu} \varepsilon_{v} \omega$. It means that polynomial forms $a \varepsilon_{\nu} \iota_{\nu} \omega$ and $-b \iota_{\nu} \varepsilon_{\nu} \omega$ are both tangential and normal on $\mathbb{R}^{n}$. Since $a, b \neq 0, \varepsilon_{\nu} \iota_{\nu} \omega=0 \iota_{\nu} \varepsilon_{\nu} \omega=0$. Formula (2.4) now implies that $\omega=0$. 
2.2. Relations between $d, \delta, \iota_{v}$ and $\varepsilon_{v}$

Let $d$ and $\delta$ denote the (exterior) differential and codifferential, respectively. Recall that for any $p$-form $\omega, d \omega$ (respectively $\delta \omega)$ is $(p+1)$-form (respectively $(p-1)$ form) which coefficients are given by ([15, pages 9-10])

$$
\begin{aligned}
& (d \omega)_{i_{0}, \ldots, i_{p}}=\partial_{i_{0}} \omega_{i_{1}, i_{2}, \ldots, i_{p}}-\partial_{i_{1}} \omega_{i_{0}, i_{2}, \ldots, i_{p}}-\cdots-\partial_{i_{p}} \omega_{i_{1}, i_{2}, \ldots, i_{0}}, \\
& (\delta \omega)_{i_{2}, \ldots, i_{p}}=-\sum_{i=1}^{n} \partial_{i} \omega_{i, i_{2}, \ldots, i_{p}} .
\end{aligned}
$$

Manifestly, $d: \Lambda_{k}^{p} \rightarrow \Lambda_{k-1}^{p+1}$ and $\delta: \Lambda_{k}^{p} \rightarrow \Lambda_{k-1}^{p-1}$. It is very-well known that $d$ is graded derivation, $d^{2}=d \circ d=0$ and $\delta^{2}=\delta \circ \delta=0$. Moreover, on $\Lambda^{p}$, $\delta=(-1)^{n(p+1)+1} \star d \star$ and $d=(-1)^{n(n-p)} \star \delta \star$.

Observe that

$$
d \varepsilon_{v}=-\varepsilon_{v} d \quad \text { and } \quad \delta \iota_{v}=-\iota_{v} \delta .
$$

Indeed. Since $d$ is graded derivation and $v^{\star}$ is closed, the first formula of (2.5) follows. To prove the second one it suffices to take $p$-form $\omega$ and compute coefficients of $\delta \iota_{v}$ and $\iota_{v} \delta$.

Proposition 2.4. Suppose $\omega$ is a ( $p / k)$-form. We have the following identities

$$
\begin{aligned}
\delta \varepsilon_{\nu} \omega & =-\varepsilon_{\nu} \delta \omega-(n-p+k) \omega, \\
d \iota_{\nu} \omega & =-\iota_{\nu} d \omega+(p+k) \omega .
\end{aligned}
$$

Proof. Let $\delta_{j}^{i}$ denote Kronecker symbol. Compute coefficients of $\varepsilon_{\nu} \delta \omega$ and $\delta \varepsilon_{\nu} \omega$.

$$
\begin{aligned}
\left(\varepsilon_{\nu} \delta \omega\right)_{i_{1}, \ldots, i_{p}}= & x^{i_{1}}(\delta \omega)_{i_{2}, i_{3} \ldots, i_{p}}-x^{i_{2}}(\delta \omega)_{i_{1}, i_{3} \ldots, i_{p}}-\cdots-x^{i_{p}}(\delta \omega)_{i_{2}, i_{3} \ldots, i_{1}} \\
= & -\sum_{i_{0}=1}^{n}\left(x^{i_{1}} \partial_{i_{0}} \omega_{i_{0}, i_{2}, i_{3} \ldots, i_{p}}-x^{i_{2}} \partial_{i_{0}} \omega_{i_{0}, i_{1}, i_{3} \ldots, i_{p}}-\cdots\right. \\
& \left.\cdots-x^{i_{p}} \partial_{i_{0}} \omega_{i_{0}, i_{2}, i_{3} \ldots, i_{1}}\right) \\
= & -\sum_{i_{0}=1}^{n}\left(x^{i_{1}} \partial_{i_{0}} \omega_{i_{0}, i_{2}, i_{3} \ldots, i_{p}}+x^{i_{2}} \partial_{i_{0}} \omega_{i_{1}, i_{0}, i_{3} \ldots, i_{p}}+\cdots\right. \\
& \left.\cdots+x^{i_{p}} \partial_{i_{0}} \omega_{i_{1}, i_{2}, i_{3} \ldots, i_{0}}\right) .
\end{aligned}
$$


On the other hand

$$
\begin{aligned}
\left(\delta \varepsilon_{v} \omega\right)_{i_{1}, \ldots, i_{p}}= & -\sum_{i_{0}=1}^{n} \partial_{i_{0}}\left(x^{i_{0}} \omega_{i_{1}, i_{2}, \ldots, i_{p}}-x^{i_{1}} \omega_{i_{0}, i_{2}, \ldots, i_{p}}-\cdots-x^{i_{p}} \omega_{i_{1}, i_{2}, \ldots, i_{0}}\right) \\
= & -\sum_{i_{0}=1}^{n}\left(\delta_{i_{0}}^{i_{0}} \omega_{i_{1}, i_{2}, \ldots, i_{p}}-\delta_{i_{0}}^{i_{1}} \omega_{i_{0}, i_{2}, \ldots, i_{p}}-\cdots-\delta_{i_{0}}^{i_{p}} \omega_{i_{1}, i_{2}, \ldots, i_{0}}\right) \\
& -\sum_{i_{0}=1}^{n}\left(x^{i_{0}} \partial_{i_{0}} \omega_{i_{1}, i_{2}, \ldots, i_{p}}-x^{i_{1}} \partial_{i_{0}} \omega_{i_{0}, i_{2}, \ldots, i_{p}}-\cdots-x^{i_{p}} \partial_{i_{0}} \omega_{i_{1}, i_{2}, \ldots, i_{0}}\right) \\
= & -(n-p+k) \omega_{i_{1}, \ldots, i_{p}} \\
& +\sum_{i_{0}=1}^{n}\left(x^{i_{1}} \partial_{i_{0}} \omega_{i_{0}, i_{2}, \ldots, i_{p}}+x^{i_{2}} \partial_{i_{0}} \omega_{i_{1}, i_{0}, \ldots, i_{p}}+\cdots+x^{i_{p}} \partial_{i_{0}} \omega_{i_{1}, i_{2}, \ldots, i_{0}}\right) .
\end{aligned}
$$

In the last equality the Euler property was used to the homogeneous polynomial $\omega_{i_{1}, \ldots, i_{p}} \in \mathcal{P}_{k}$.

Making similar computations one can obtain the second formula. One can also use the well-known identity (see [5, Theorem 7.9(2)]) : $d \iota_{v}+\iota_{v} d=\mathcal{L}_{v}$, where $\mathcal{L}_{v}$ is Lie derivative in the direction of $v$.

As a consequence of (2.5), (2.4) and Proposition 2.4 we obtain:

Proposition 2.5. For any polynomial form $\omega$ we have

$$
\begin{aligned}
& d\left(r^{2} \omega\right)=r^{2} d \omega+2 \varepsilon_{\nu} \omega \\
& \delta\left(r^{2} \omega\right)=r^{2} \delta \omega-2 \iota_{\nu} \omega .
\end{aligned}
$$

Remark 2.6. Proposition 2.5 holds for any differential form $\omega$, not necessary polynomial.

Consider now $d$ and $\delta$ as operators $d: \Lambda_{k}^{p} \rightarrow \Lambda_{k-1}^{p+1}$ and $\delta: \Lambda_{k}^{p} \rightarrow \Lambda_{k-1}^{p-1}$. Let $d^{\star}$ and $\delta^{\star}$ denote adjoint operator, respectively. One of the most important tool, we will use is:

Theorem 2.7. For any $(p / k)$-form $\omega, \delta^{\star} \omega=-\varepsilon_{\nu} \omega$ and $d^{\star} \omega=\iota_{\nu} \omega$.

Proof. We will prove only the first formula of our assertion. The second one can be obtained in a similar way. 
Fix $p, k>0$. To prove the first formula take $\omega \in \Lambda_{k}^{p}$ and $\eta \in \Lambda_{k-1}^{p-1}$. We have

$$
\begin{aligned}
(\delta \omega \mid \eta) & =\frac{1}{(p-1) !} \sum_{i_{2}, \ldots, i_{p}=1}^{n}\left((\delta \omega)_{i_{2}, \ldots, i_{p}}, \eta_{i_{2}, \ldots, i_{p}}\right) \\
& =-\frac{1}{(p-1) !} \sum_{i_{2}, \ldots, i_{p}=1}^{n}\left(\sum_{i_{1}=1}^{n} \partial_{i_{1}} \omega_{i_{1}, i_{2}, \ldots, i_{p}}, \eta_{i_{1}, i_{2}, \ldots, i_{p}}\right) \\
& =-\frac{1}{(p-1) !} \sum_{i_{1}, i_{2}, \ldots, i_{p}=1}^{n}\left(\omega_{i_{1}, i_{2}, \ldots, i_{p}}, x^{i_{1}} \eta_{i_{2}, \ldots, i_{p}}\right) .
\end{aligned}
$$

On the other hand

$$
\begin{aligned}
& \left(\omega \mid \varepsilon_{\nu} \eta\right)=\frac{1}{p !} \sum_{i_{1}, \ldots, i_{p}=1}^{n}\left(\omega_{i_{1}, \ldots, i_{p}},\left(\varepsilon_{\nu} \eta\right)_{i_{1}, \ldots, i_{p}}\right)
\end{aligned}
$$

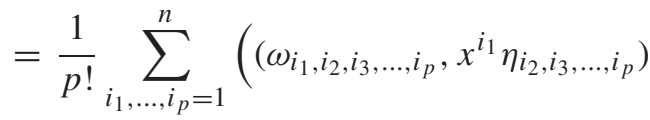

$$
\begin{aligned}
& \left.-\left(\omega_{i_{1}, i_{2}, i_{3}, \ldots, i_{p}}, x^{i_{2}} \eta_{i_{1}, i_{3}, \ldots, i_{p}}\right)-\cdots-\left(\omega_{i_{1}, i_{2}, i_{3}, \ldots, i_{p}}, x^{i_{p}} \eta_{i_{2}, i_{3}, \ldots, i_{1}}\right)\right)
\end{aligned}
$$

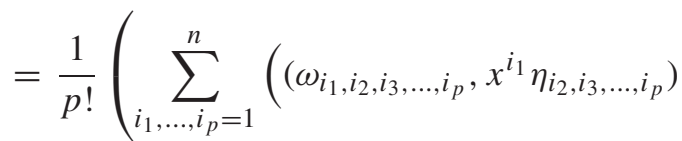

$$
\begin{aligned}
& +\sum_{i_{1}, \ldots, i_{p}=1}^{n}\left(\omega_{i_{1}, i_{2}, i_{3}, \ldots, i_{p}}, x^{i_{1}} \eta_{i_{2}, i_{3}, \ldots, i_{p}}\right) \\
& +\cdots \\
& \left.\left.+\sum_{i_{1}, i_{2}, i_{3}, \ldots, i_{p}=1}^{n} \omega_{i_{1}, i_{2}, i_{3}, \ldots, i_{p}}, x^{i_{1}} \eta_{i_{2}, i_{3}, \ldots, i_{p}}\right)\right) \\
& =\frac{1}{p !} p \sum_{i_{1}, i_{2}, \ldots, i_{p}=1}^{n}\left(\omega_{i_{1}, i_{2}, \ldots, i_{p}}, x^{i_{1}} \eta_{i_{2}, \ldots, i_{p}}\right) \\
& =\frac{1}{(p-1) !} \sum_{i_{1}, i_{2}, \ldots, i_{p}=1}^{n}\left(\omega_{i_{1}, i_{2}, \ldots, i_{p}}, x^{i_{1}} \eta_{i_{2}, \ldots, i_{p}}\right) \text {. }
\end{aligned}
$$

Therefore, the first formula follows. 


\section{Laplace type operators}

\subsection{Definition and basic properties of Laplace type operator}

Let $a, b>0$. Differential operator

$$
L=L_{a, b}=a d \delta+b \delta d
$$

is called the Laplace type operator. In particular, $L_{1,1}$ is just the Laplace-Beltrami operator $\Delta=d \delta+\delta d$. Moreover, $S^{\star} S=(n-1) / n d \delta+1 / 2 \delta d$, considered as an operator $\Lambda^{1} \rightarrow \Lambda^{1}$, is called the Ahlfors-Laplace operator (cf. [10]).

Take any $p$-form $\omega$ and compute coefficients the of $d \delta \omega$ and $\delta d \omega$. We have

$$
\begin{aligned}
(d \delta \omega)_{i_{1}, \ldots, i_{p}}= & -\sum_{i=1}^{n}\left(\partial_{i_{1}, i}^{2} \omega_{i, i_{2}, i_{3}, \ldots, i_{p}}+\partial_{i_{2}, i}^{2} \omega_{i_{1}, i, i_{3}, \ldots, i_{p}}+\cdots+\partial_{i_{p}, i}^{2} \omega_{i_{1}, i_{2}, i_{3}, \ldots, i}\right), \\
(\delta d \omega)_{i_{1}, \ldots, i_{p}}= & -\sum_{i=1}^{n} \partial_{i, i}^{2} \omega_{i_{1}, \ldots, i_{p}} \\
& +\sum_{i=1}^{n}\left(\partial_{i_{1}, i}^{2} \omega_{i, i_{2}, i_{3}, \ldots, i_{p}}+\partial_{i_{2}, i}^{2} \omega_{i_{1}, i, i_{3}, \ldots, i_{p}}+\cdots+\partial_{i_{p}, i}^{2} \omega_{i, i_{2}, i_{3}, \ldots, i}\right),
\end{aligned}
$$

Therefore, for any $f \in \mathcal{P}_{k},(d \delta+\delta d) f=\delta d f=-r^{2}(D) f$. It means that, in the case of 0 -forms, the Laplace-Beltrami operator $L_{1,1}$ and classical Laplace operator coincide. Moreover, for any $p$-form $\omega$,

$$
(\Delta \omega)_{i_{1}, \ldots, i_{p}}=\Delta \omega_{i_{1}, \ldots, i_{p}} .
$$

Because of this and (2.1) we obtain that for any $\omega \in \Lambda_{k}^{p}$,

$$
\Delta\left(r^{2} \omega\right)=-2(n+2 k) \omega+r^{2} \Delta \omega .
$$

It is clear that $L$ maps $\Lambda_{k}^{p}$ into $\Lambda_{k-2}^{p}$. Let denote

$$
\begin{aligned}
\mathfrak{L}_{k}^{p} & =\operatorname{kernel} \text { of }\left(L: \Lambda_{k}^{p} \rightarrow \Lambda_{k-2}^{p}\right), \\
\mathfrak{H}_{k}^{p} & =\operatorname{kernel} \text { of }\left(\Delta: \Lambda_{k}^{p} \rightarrow \Lambda_{k-2}^{p}\right) .
\end{aligned}
$$

In particular, (3.1) implies that ( $p / k$ )-form $\omega$ is harmonic, i.e, $\Delta \omega=0$, if and only if each coefficient $\omega_{i_{1}, \ldots, i_{p}}$ of $\omega$ is a member of $\mathcal{H}_{k}$. Thus, $\operatorname{dim} \mathfrak{H}_{k}^{p}=\left(\begin{array}{l}n \\ p\end{array}\right) \operatorname{dim} \mathcal{H}_{k}=$ $\operatorname{dim} \Lambda_{k}^{p}-\operatorname{dim} \Lambda_{k-2}^{p}$. As a direct consequence of (3.1) and Proposition 2.1 we obtain:

Corollary 3.1. For each $\omega \in \Lambda_{k}^{p}$ there exist unique polynomial forms $\eta_{j} \in \mathfrak{H}_{k-2 j}^{p}$, $0 \leq j \leq m_{k}=[k / 2]$ such that $\omega=\eta_{0}+r^{2} \eta_{1}+\cdots+\left(r^{2}\right)^{m_{k}} \eta_{m_{k}}$. 
Using (2.5) and Proposition 2.4 we get, that for any polynomial form $\omega$

$$
\iota_{\nu} \Delta \omega=\Delta \iota_{\nu} \omega-2 \delta \omega .
$$

Consider $L=L_{a, b}$ as an operator $L: \Lambda_{k}^{p} \rightarrow \Lambda_{k-2}^{p}$. Clearly, if $k=0,1$ then $\Lambda_{k}^{p}=\mathfrak{L}_{k}^{p}=\mathfrak{H}_{k}^{p}$. Moreover, $\mathfrak{L}_{k}^{0}=\mathcal{H}_{k}$ and $\mathfrak{L}_{k}^{n}$ is in a natural way isomorphic to $\mathcal{H}_{k}$.

Let $\mathfrak{l}=\mathfrak{l}_{a, b}=a \varepsilon_{v} \iota_{v}+b \iota_{v} \varepsilon_{v}$ and let $L_{a, b}^{\star}$ denote the adjoint operator to $L_{a, b}$. As a direct consequence of Theorem 2.7 we get that for any $(p / k)$-form $\omega$,

$$
L_{a, b}^{\star}(\omega)=-\mathfrak{l}_{a, b}(\omega) .
$$

In particular, $\Delta^{\star} \omega=-r^{2} \omega$, for $\mathfrak{l}_{1,1} \omega=r^{2} \omega$. From (3.4) we have

$$
\Lambda_{k}^{p}=\mathfrak{L}_{k}^{p} \oplus^{\perp} \operatorname{iml}
$$

Since $a, b>0$, Proposition 2.3 implies that $\mathfrak{l}_{a, b}$ is one-to-one. Therefore, $\operatorname{dim} \mathfrak{L}_{k}^{p}=$ $\operatorname{dim} \Lambda_{k}^{p}-\operatorname{dim} \Lambda_{k-2}^{p}=\operatorname{dim} \mathfrak{H}_{k}^{p}$.

The next part of this section has pure technical character. We will introduce some special linear operator $I_{c}$ and derive the compositions $L \circ I_{c}$ and $\Delta \circ I_{c}$. This knowledge became useful in the Section 3.3, where we decompose $\mathfrak{L}_{k}^{p}$ onto direct sum of four mutually orthogonal subspaces. One of them will be described as an image on $I_{c}$, going one step future.

Take any $\eta \in \Lambda_{l}^{q}$ then $d \eta \in \Lambda_{l-1}^{q+1}$. Using Proposition 2.5 and 2.4 one can easily obtain

$$
\begin{aligned}
& d \delta\left(r^{2} d \eta\right)=-2(q+l) d \eta+2 \varepsilon_{\nu} \delta(d \eta)+r^{2} d \delta(d \eta), \\
& \delta d\left(r^{2} d \eta\right)=-2(n-q+l-2) d \eta-2 \varepsilon_{\nu} \delta(d \eta) .
\end{aligned}
$$

Therefore,

$$
L\left(r^{2} d \eta\right)=-2(a(q+l)+b(n-q+l-2)) d \eta+2(a-b) \varepsilon_{\nu} \delta(d \eta)+r^{2} L(d \eta) .
$$

Using (2.5) and Proposition 2.4 one can check that

$$
L\left(\varepsilon_{v} \eta\right)=(b(n-q+l-2)-a(n-q+l)) d \eta+\varepsilon_{v}(L \eta),
$$

In particular, if $L=\Delta$, i.e., $a=b=1$, for any $\eta \in \Lambda_{l}^{q}$ we get

$$
\begin{aligned}
\Delta\left(r^{2} d \eta\right) & =-2(n+2 l-2) d \eta+r^{2} \Delta(d \eta), \\
\Delta\left(\varepsilon_{\nu} \eta\right) & =-2 d \eta+\varepsilon_{\nu}(\Delta \eta) .
\end{aligned}
$$

For any constant $c \in \mathbb{R}$ we define a linear operator

$$
I_{c}=I_{c}(q+1, l+1)=\varepsilon_{v}-c r^{2} d: \Lambda_{l}^{q} \rightarrow \Lambda_{l+1}^{q+1} .
$$


As a straightforward consequence of above considerations we obtain that for any $(q / l)$-form $\eta$,

$$
\begin{aligned}
L\left(I_{c} \eta\right)= & ((b-a)(n-q+l) \\
& +2 c(a(q+l)+b(n-q+l-2))-2 b) d \eta \\
& -2 c(a-b) \varepsilon_{\nu} \delta d \eta-c r^{2} L(d \eta)+\varepsilon_{\nu}(L \eta), \\
\Delta\left(I_{c} \eta\right)= & (2 c(n+2 l-2)-2) d \eta-c r^{2} \Delta(d \eta)+\varepsilon_{\nu}(\Delta \eta) .
\end{aligned}
$$

3.2. Spaces $\chi_{p, k}$ and $\chi_{p, k}^{0}$

For any $0 \leq p \leq n$ and $k \geq 0$ define

$$
\chi_{p, k}=\mathfrak{H}_{k}^{p} \cap \operatorname{ker} \delta \text {, and } \chi_{p, k}^{0}=\chi_{p, k} \cap \operatorname{ker} \iota_{v} .
$$

Put $\chi_{q, l}=\chi_{q, l}^{0}=\{0\}$ if either $q<0$ or $l<0$. Manifestly, $\mathfrak{H}_{k}^{0}=\chi_{0, k}=\chi_{0, k}^{0}=\mathcal{H}_{k}$ and $\mathfrak{H}_{0}^{p}=\Lambda_{0}^{p}$.

The spaces $\chi_{p, k}^{0}$ 's will play a fundamental role in the next part of this paper. In the next section we will build a whole decomposition of $\mathfrak{L}_{k}^{p}$ using that spaces. Now we show some basic properties of $\chi_{p, k}^{0}$ and operators $d, \varepsilon_{v} d$ and $I_{c}$ restricted to it.

Let $\omega \in \chi_{p, 0}^{0}$ then $\iota_{\nu} \omega=0$ and $d \omega=0$. By Proposition 2.4 we obtain $0=d \iota_{\nu} \omega=-\iota_{\nu} d \omega+(p+0) \omega$. So

$$
\chi_{p, 0}^{0}=\{0\} \quad \text { if } p>0
$$

Observe that $d \chi_{p-1, k+1} \subset \chi_{p, k}$. On the other hand, using (2.5) and (3.3) we check that $\iota_{\nu} \chi_{p, k} \subset \chi_{p-1, k+1}$. Since $\iota_{v}^{2}=0$ we even have $\iota_{v}: \chi_{p, k} \rightarrow \chi_{p-1, k+1}^{0}$.

Theorem 2.7 implies now that $\chi_{p, k}=\chi_{p, k}^{0} \oplus^{\perp} d \chi_{p-1, k+1}$. Since $d^{2}=0$, for any $0 \leq p \leq n$ and $k \geq 0$ we have

$$
\chi_{p, k}=\chi_{p, k}^{0} \oplus^{\perp} d \chi_{p-1, k+1}^{0}
$$

Moreover, as a direct consequence of (3.6) we get

$$
\varepsilon_{\nu} d \chi_{p-2, k}^{0} \subset \mathfrak{H}_{k}^{p}
$$

By Proposition 2.4 for $\eta \in \chi_{p-2, k}^{0}$.

$$
\delta \varepsilon_{\nu} d \eta=-(n-p+k) d \eta,
$$

Therefore,

$$
\delta: \varepsilon_{\nu} d \chi_{p-2, k}^{0} \rightarrow d \chi_{p-2, k}^{0} .
$$


Summarizing one can prove the following:

\section{Proposition 3.2.}

(i) If $0<p \leq n$ and $k \geq 0$ then $d: \chi_{p-1, k+1}^{0} \rightarrow \chi_{p, k}$ is one-to-one and for any $\eta \in \chi_{p-1, k+1}^{0},(d \eta \mid d \eta)=(p+k)(\eta \mid \eta)$.

(ii) If $0<p \leq n$ and $k \geq 0$ then $\iota_{v}: d \chi_{p-1, k+1}^{0} \rightarrow \chi_{p-1, k+1}^{0}$ is a bijection and for any $\eta \in \chi_{p-1, k+1}^{0},\left(\iota_{\nu} d \eta \mid \iota_{\nu} d \eta\right)=(p+k)(d \eta \mid d \eta)$.

(iii) If $2 \leq p \leq n$ and $k \geq 0$ then $\varepsilon_{v}: d \chi_{p-2, k}^{0} \rightarrow \mathfrak{H}_{k}^{p}$ is one-to-one and for any $\eta \in \chi_{p-2, k}^{0},\left(\varepsilon_{\nu} d \eta \mid \varepsilon_{v} d \eta\right)=(n-p+k)(d \eta \mid d \eta)$.

(iv) If $2 \leq p \leq n$ and $k \geq 0$ then $\delta: \varepsilon_{\nu} d \chi_{p-2, k}^{0} \rightarrow d \chi_{p-2, k}^{0}$ is a bijection and for any $\eta \in \chi_{p-2, k}^{0},\left(\delta \varepsilon_{\nu} d \eta \mid \delta \varepsilon_{\nu} d \eta\right)=(n-p+k)^{2}(d \eta \mid d \eta)$.

For $c \in \mathbb{R}$ let

$$
I_{c}=I_{c}(p, k)=\varepsilon_{v}-c r^{2} d: \Lambda_{k-1}^{p-1} \rightarrow \Lambda_{k}^{p}
$$

Take any $\eta \in \chi_{p-1, k-1}$ then $\delta d \eta=0$. By Proposition 2.4 and 2.5 we obtain

$$
\left(\delta I_{c}\right) \eta=-(n-p+k) \eta+2 c \iota_{\nu} d \eta
$$

Remark 3.3. Observe that if $\eta=d \eta^{\prime}, \eta^{\prime} \in \chi_{p-2, k}$ then $I_{c} \eta=\varepsilon_{\nu} d \eta^{\prime}$ and (3.13) reduces to (3.12).

Since $\delta \Delta=\Delta \delta,(3.8)$ implies that $\delta I_{c}\left(\chi_{p-1, k-1}\right) \subset \mathfrak{H}_{k-1}^{p-1}$. Since $\delta^{2}=0$, we conclude that

$$
\delta I_{c}: \chi_{p-1, k-1} \rightarrow \chi_{p-1, k-1}
$$

Lemma 3.4. Suppose that $1 \leq p \leq n$ and $k \geq 1$. Let $c \in \mathbb{R}$ and $c_{0}=\frac{1}{2} \frac{n-p+k}{p+k-2}$. If $k=1$, or $k \geq 2$ and $c \neq c_{0}$ then $I_{c}: \chi_{p-1, k-1} \rightarrow \Lambda_{k}^{p}$ is one-to-one, whereas $\delta: I_{c}\left(\chi_{p-1, k-1}\right) \rightarrow \chi_{p-1, k-1}$ is a surjective. Moreover, for any $\eta \in \chi_{p-1, k-1}^{0}$, $\left(I_{c} \eta \mid I_{c} \eta\right)=\rho(c)(\eta \mid \eta)$, where

$$
\rho(c)=2(n+2 k-4)(p+k-2) c^{2}-4(p+k-2) c+(n-p+k) .
$$

Proof. It suffices to show that $\delta I_{c}: \chi_{p-1, k-1} \rightarrow \chi_{p-1, k-1}$ is a bijection. Take $\eta \in \chi_{p-1, k-1}$ and suppose that $\delta I_{c} \eta=0$.

If $k=1, d \eta=0$. Formula (3.13) implies that $0=\left(\delta I_{c}\right) \eta=-(n-p+1) \eta$. Since $(n-p+1) \neq 0, \eta=0$.

Let $k \geq 2$. We have $d\left(\delta I_{c}\right) \eta=0$, for $\delta I_{c} \eta=0$. By (3.13) and Proposition 2.4, $(2 c(p+k-2)-(n-p+k)) d \eta=0$. Since $c \neq c_{0}, \eta$ must be closed. Therefore, $0=\left(\delta I_{c}\right) \eta=-(n-p+k) \eta$. Since $n-p+k>0, \eta=0$. 
Take any $\omega^{\prime}=I_{c}\left(\eta^{\prime}\right)$, where $\eta^{\prime} \in \chi_{p-1, k-1}^{0}$ and $\omega^{\prime \prime}=\varepsilon_{\nu} d \eta^{\prime \prime}$, where $\eta^{\prime \prime} \in$ $\chi_{p-2, k}^{0}$. Using Theorem 2.7, Proposition 2.4 and (3.11) we get

$$
\begin{aligned}
\left(\omega^{\prime} \mid \omega^{\prime \prime}\right) & =\left(\varepsilon_{\nu} \eta^{\prime}-c r^{2} d \eta^{\prime} \mid \varepsilon_{v} d \eta^{\prime \prime}\right) \\
& =\left(\varepsilon_{v} \delta \eta^{\prime}+(n-p+k) \eta^{\prime} \mid d \eta^{\prime \prime}\right)+c\left(d \eta^{\prime} \mid \Delta \varepsilon_{v} d \eta^{\prime \prime}\right) \\
& =(n-p+k)\left(\iota_{\nu} \eta^{\prime} \mid \eta^{\prime \prime}\right) \\
& =0 .
\end{aligned}
$$

Therefore, we obtain the following decomposition

$$
I_{c}\left(\chi_{p-1, k-1}\right)=I_{c}\left(\chi_{p-1, k-1}^{0}\right) \oplus^{\perp} \varepsilon_{\nu} d \chi_{p-2, k}^{0} .
$$

Remark 3.5. As we will see in the next section spaces $\chi_{p, k}$ and $I_{c}\left(\chi_{p . k}\right)$ are mutually orthogonal. Therefore, in the light of (3.10) and (3.14), the algebraic sum $V_{k}^{p}(c)=\chi_{p, k}+I_{c}\left(\chi_{p, k}\right)$ splits as $V_{k}^{p}(c)=\chi_{p, k}^{0} \oplus^{\perp} d \chi_{p-1, k+1}^{0} \oplus^{\perp} \varepsilon_{\nu} d \chi_{p-2, k}^{0} \oplus^{\perp}$ $I_{c}\left(\chi_{p-1, k-1}^{0}\right)$, where only the last component depends on $c$, whereas three remaining components are subspaces of $\mathfrak{H}_{k}^{p}$ (even of ker $L_{a, b}$, for any $a, b>0$ ). In the next section we will show that the kernel of any Laplace type operator is of form $V_{k}^{p}(c)$ for some $c$.

\subsection{Decomposition of $\mathfrak{L}_{k}^{p}$}

Let $L=L_{a, b}=a d \delta+b \delta d, a, b>0$. Since for any $\omega \in \chi_{p, k} \delta d \omega=0$, $\chi_{p, k}^{0} \subset \chi_{p, k} \subset \mathfrak{L}_{k}^{p}$. Suppose that $L \omega=0$, then $d \delta \omega=-(b / a) \delta d \omega$, so $\Delta \delta \omega=0$. Hence $\delta \mathfrak{L}_{k}^{p} \subset \chi_{p-1, k-1}$. In particular, $\delta \mathfrak{H}_{k}^{p} \subset \chi_{p-1, k-1}$. Put

$$
c_{L}=c_{L}(p, k)= \begin{cases}\frac{1}{2} \frac{2 b-(b-a)(n-p+k)}{a(p+k-2)+b(n-p+k-2)}, & \text { if } k \geq 2,0<p \leq n, \\ 0, & \text { otherwise }\end{cases}
$$

Notice that our assumption $(a, b>0$ and $n \geq 2)$ ensure that $c_{L}$ is well-defined. In the particular case,

$$
c_{\Delta}=c_{\Delta}(p, k)= \begin{cases}\frac{1}{n+2 k-4}, & \text { if } k \geq 2,0<p \leq n, \\ 0, & \text { otherwise. }\end{cases}
$$

Remark 3.6. Observe that the constant $c_{\Delta}$ do not depend on $p$.

Let, as in previously,

$$
I_{c}=I_{c}(p, k)=\varepsilon_{v}-c r^{2} d: \Lambda_{k-1}^{p-1} \rightarrow \Lambda_{k}^{p} .
$$

Put, for simplicity

$$
I_{L}=I_{L}(p, k)=I_{c_{L}}=I_{c_{L}(p, k)}(p \cdot k) .
$$


A goal of this section is to prove the following:

Theorem 3.7. For any $0 \leq p \leq n$ and $k \geq 0$ the space $\mathfrak{L}_{k}^{p}$ is the direct sum offour mutually orthogonal subspaces:

$$
\mathfrak{L}_{k}^{p}=\chi_{p, k}^{0} \oplus^{\perp} d \chi_{p-1, k+1}^{0} \oplus^{\perp} \varepsilon_{\nu} d \chi_{p-2, k}^{0} \oplus^{\perp} I_{L}(p, k) \chi_{p-1, k-1}^{0} .
$$

Moreover, $\chi_{p, k}^{0}, d \chi_{p-1, k+1}^{0}$ and $\varepsilon_{\nu} d \chi_{p-2, k}^{0}$ are subspaces of $\mathfrak{H}_{k}^{p}$.

In the special case we get

$$
\mathfrak{H}_{k}^{p}=\chi_{p, k}^{0} \oplus^{\perp} d \chi_{p-1, k+1}^{0} \oplus^{\perp} \varepsilon_{\nu} d \chi_{p-2, k}^{0} \oplus^{\perp} I_{\Delta}(p, k) \chi_{p-1, k-1}^{0} .
$$

Remark 3.8. It is worth to say that in (3.16) some subspaces may equals $\{0\}$ (see 3.9). In particular, if $p=1$ then $\varepsilon_{\nu} d \chi_{-1, k}=\{0\}$, so $\mathfrak{L}_{k}^{1}=\chi_{1, k}^{0} \oplus^{\perp} d \chi_{0, k+1}^{0} \oplus^{\perp}$ $I_{L} \chi_{0, k-1}^{0}=\chi_{1, k}^{0} \oplus^{\perp} d \mathcal{H}_{k+1} \oplus^{\perp} I_{L}\left(\mathcal{H}_{k-1}\right)$.

To prove Theorem 3.7 it suffices to apply the following: Lemma 3.9, Corollary 3.13 and Lemma 3.14 below.

Lemma 3.9. If either $p=0$ or $k=0$ then the decomposition (3.16) holds.

Proof. If $p=0$ our assertion is a direct consequence of the following equalities $\mathfrak{H}_{k}^{0}=\chi_{0, k}=\chi_{0, k}^{0}=\mathcal{H}_{k}$ and $\mathfrak{L}_{k}^{0}=\mathcal{H}_{k}$.

Consider the case $p>0$ and $k=0$. Then $\mathfrak{H}_{0}^{p}=\Lambda_{0}^{p}, \varepsilon_{\nu} d \chi_{p-2,0}^{0}=I_{L} \chi_{p-1,-1}^{0}=$ $\{0\}$, and $\chi_{p, 0}^{0}=\{0\}$ (see (3.9)). To prove (3.16) it suffices to show that $\mathfrak{H}_{0}^{p}=$ $d \chi_{p-1,1}^{0}$. To do this take $\omega \in \mathfrak{H}_{0}^{p}$ and put $\eta=(1 / p) \iota_{\nu} \omega$. Clearly, $\iota_{\nu} \eta=0, \delta \eta=0$ and $\Delta \eta=0($ see $(3.3))$, so $\eta \in \chi_{p-1,1}^{0}$. One can easily check that $d \eta=\omega$.

Suppose now that $0<p \leq n$ and $k>0$. Identity (3.7) implies that for any $\eta \in \chi_{p-1, k-1}$

$$
L\left(I_{c} \eta\right)=((b-a)(n-p+k)-2 b+2 c(a(p+k-2)+b(n-p+k-2)) d \eta .
$$

Thus we obtain $I_{L}\left(\chi_{p-1, k-1}\right) \subset \mathfrak{L}_{k}^{p}$. Hence (see (3.14)), $\varepsilon_{\nu} d \chi_{p-2, k}^{0} \subset \mathfrak{L}_{k}^{p}$.

Lemma 3.10. Suppose that $k>0$ and $0<p \leq n$.

(i) Spaces $\chi_{p, k}$ and $I_{L}\left(\chi_{p-1, k-1}\right)$ are mutually orthogonal.

(ii) If either $k \neq 2$ or $p<n$ then $I_{c_{L}}: \chi_{p-1, k-1} \rightarrow \mathfrak{L}_{k}^{p}$ is one-to-one and $\delta: I_{c_{L}}\left(\chi_{p-1, k-1}\right) \rightarrow \chi_{p-1, k-1}$ is a bijection.

(iii) If either $k \neq 2$ or $p<n$ then $\mathfrak{L}_{k}^{p}=\chi_{p, k} \oplus^{\perp} I_{L}\left(\chi_{p-1, k-1}\right)$. 
Proof. (i) Take $\omega \in \chi_{p, k}$ and $\eta \in \chi_{p-1, k-1}$. Using Theorem 2.7 and (3.4) we obtain

$$
\left(\omega \mid \varepsilon_{v} \eta-c_{L} r^{2} d \eta\right)=-(\delta \omega \mid \eta)+c_{L}(\Delta \omega \mid d \eta)=0 .
$$

Therefore (i) follows.

(ii) If $k=1$ then our assertion is a direct consequence of Lemma 3.4. Therefore, we may assume that $k \geq 2$, and either $k>2$ or $p<n$. The assumption implies immediately that

$$
n \neq-2(k-1) \text { and } n \neq-(k-p-2) \text {. }
$$

Lemma 3.4 implies that it suffices to show that $c_{L} \neq c_{0}$. Suppose that $c_{L}=$ $c_{0}$. One easily checks that the equation $c_{L}=c_{0}$ is equivalent to the equation $(n+k-p-2)(n+2 k-2)=0$. It contradicts $(3.18)$.

(iii) Relations $I_{L}\left(\chi_{p-1, k-1}\right) \subset \mathfrak{L}_{k}^{p}, \delta \mathfrak{L}_{k}^{p} \subset \chi_{p-1, k-1}$, and point (ii) implies that $\delta: \mathfrak{L}_{k}^{p} \rightarrow \chi_{p-1, k-1}$ is a surjection. Thus $\operatorname{dim} \mathfrak{L}_{k}^{p}=\operatorname{dim} \chi_{p, k}+\operatorname{dim} \chi_{p-1, k-1}=$ $\operatorname{dim} \chi_{p, k}+\operatorname{dim} I_{L}\left(\chi_{p-1, k-1}\right)$. Since $\chi_{p, k}$ and $I_{L}\left(\chi_{p-1, k-1}\right)$ are mutually orthogonal subspaces of $\mathfrak{L}_{k}^{p}$ point (iii) follows.

Corollary 3.11. If $k>0$ and $0<p<n$ then $I_{L}: \chi_{p-1, k-1} \rightarrow \mathfrak{L}_{k}^{p}$ is one-to-one, moreover we have the decomposition $\mathfrak{L}_{k}^{p}=\chi_{p, k} \oplus^{\perp} I_{L}\left(\chi_{p-1, k-1}\right)$.

Remark 3.12. Corollary 3.11 is the sufficiently tool in solving Dirichlet boundary problem for the Laplace type operator.

As a direct consequence of (3.10), (3.14) and Lemma 3.10 we obtain:

Corollary 3.13. Suppose that $k>0$ and $0<p \leq n$. If either $k \neq 2$ or $p<n$ then $\mathfrak{L}_{k}^{p}$ has the decomposition (3.16).

\section{Lemma 3.14.}

(i) If $p=n$ and $k=2$ then $I_{L}\left(\chi_{n-1,1}^{0}\right)=\{0\}$.

(ii) The map $\delta: \mathfrak{H}_{2}^{n} \rightarrow d \chi_{n-2,2}^{0}$ is a surjection.

(iii) $\mathfrak{L}_{2}^{n}=\chi_{n, 2}^{0} \oplus^{\perp} d \chi_{n-1,3}^{0} \oplus^{\perp} \varepsilon_{\nu} d \chi_{n-2,2}^{0}$.

Proof. (i) We see (cf. 3.15) that in the case $p=n$ and $k=2, c_{L}=1 / n$. Take any $\eta \in \chi_{n-1,1}^{0}$ then clearly $\varepsilon_{\nu} d \eta=0$. Thus using using (2.4) and Proposition 2.4 we obtain $r^{2} d \eta=\varepsilon_{\nu} \iota_{\nu} d \eta=\varepsilon_{\nu}\left(-d \iota_{\nu} \eta+((n-1)+1) \eta\right)$. Therefore we have $I_{c_{L}} \eta=I_{\frac{1}{n}} \eta=\varepsilon_{\nu} \eta-n^{-1} n \varepsilon_{\nu} \eta=0$.

(ii) We know that $\varepsilon_{\nu} d \chi_{n-2,2}^{0} \subset \mathfrak{H}_{2}^{n}$ (see (3.11)) and $\delta\left(\mathfrak{H}_{2}^{n}\right) \subset \chi_{n-1,1}$. Identity (3.12) implies now that $d \chi_{n-2,2}^{0} \subset \delta\left(\mathfrak{H}_{2}^{n}\right)$. Now it suffices to show that $\delta\left(\mathfrak{H}_{2}^{n}\right) \cap$ $\chi_{n-1,1}^{0}=\{0\}$. Let $\omega \in \mathfrak{H}_{2}^{n}$ and suppose that $\delta \omega \in \chi_{n-1,1}^{0}$ then $\iota_{\nu} \delta \omega=0$. Since $\omega$ is $n$-form $d \delta \omega=\Delta \omega=0$. Thus we have $0=d \iota_{\nu} \delta \omega=-\iota_{\nu} d \delta \omega+n \delta \omega=n \delta \omega$. Hence $\delta \omega=0$. 
(iii) Clearly, $\mathfrak{L}_{2}^{n}=\mathfrak{H}_{2}^{n}$. We know that $\varepsilon_{v}: d \chi_{n-2,2}^{0} \rightarrow \mathfrak{H}_{2}^{n}$ is one-to-one (see Proposition 3.2 (iii)). (ii) implies now $\operatorname{dim} \mathfrak{L}_{2}^{n}=\operatorname{dim} \chi_{n, 2}+\operatorname{dim} d \chi_{n-2,2}^{0}=$ $\operatorname{dim} \chi_{n, 2}+\operatorname{dim} \varepsilon_{v} d\left(\chi_{n-2,2}^{0}\right)$. Since $\chi_{n, 2}$ and $\varepsilon_{\nu} d \chi_{n-2,2}^{0}$ are orthogonal subspaces of $\mathfrak{L}_{2}^{n}$ (see Lemma 3.10 (i) and (3.14)) and $\chi_{n, 2}=\chi_{n, 2}^{0} \oplus^{\perp} d \chi_{n-1,3}^{0}$, (iii) follows.

Remark 3.15. It is worth to see that if operators $L^{(1)}=L_{a, b}$ and $L^{(2)}=L_{1 / b, 1 / a}$ then $c_{L^{(1)}}=c_{L^{(2)}}$. Hence $\operatorname{ker} L^{(1)}$ and $\operatorname{ker} L^{(2)}$ have the same decomposition.

The following corollary shows that the decomposition (3.17) of $\mathfrak{H}_{k}^{p}$ is complete from the the differential and co-differential point of view. A proof based on Proposition 3.2, Proposition 2.4, (3.9) and Lemma 3.14 (i) is left to the reader.

Corollary 3.16. Suppose that $\omega \in \mathfrak{H}_{k}^{p}, k>0$ and $\omega \neq 0$.

- If $\omega \in \chi_{p, k}^{0}$ then $\omega$ is not closed $(d \omega \neq 0)$ but is co-closed $(\delta \omega=0)$.

- If $\omega \in d \chi_{p-1, k+1}^{0}$ then $\omega$ is both closed and co-closed.

- If $\omega \in \varepsilon_{\nu} d \chi_{p-2, k}^{0}$ then $\omega$ is closed but not co-closed.

- If $\omega \in I_{\Delta} \chi_{p-1, k-1}^{0}$ then $\omega$ is not closed and not co-closed if $k>1$ and closed but not co-closed if $k=1$.

If $k=0$ then each $\omega \in \mathfrak{H}_{k}^{p}$ is both closed and co-closed. Moreover, in this case $\mathfrak{H}_{0}^{p}=\chi_{0,0}^{0}=\mathbb{R}$ if $p=0$ and $\mathfrak{H}_{0}^{p}=d \chi_{p-1,1}^{0}$ if $p>0$.

Remark 3.17. (1) If $p>0, d: \chi_{p-1,1}^{0} \rightarrow \mathfrak{H}_{0}^{p}$ is a bijection, thus $\operatorname{dim} \chi_{p-1,1}^{0}=$ $\operatorname{dim} \mathfrak{H}_{0}^{p}=\left(\begin{array}{l}n \\ p\end{array}\right)$. Since, $p$-forms $\iota_{\nu} \omega(I)$, where $\omega(I)=d x^{i_{1}} \wedge \cdots \wedge d x^{i_{p}}, I=$ $\left(i_{1}, \ldots, i_{p}\right), i_{1}<\cdots<i_{p}$ consist a basis of $\chi_{p-1,1}^{0}$.

(2) We know by (3.9) that $\chi_{p, 0}^{0}=\{0\}$ for $p>0$. We show now that $\chi_{n-1, k}^{0}=$ $\{0\}$ if $k \neq 1$. Indeed. Take $\eta \in \chi_{n-1, k}^{0}$ and put $\omega=d \eta \in \mathfrak{H}_{k-1}^{n}$. Then $\delta \omega=$ $-d \delta \eta=0$ and $\varepsilon_{v} \omega=0$, for $\omega$ is a $n$-form. Thus, we have $0=(\delta \omega \mid \delta \omega)=$ $-\left(\omega \mid \varepsilon_{\nu} \delta \omega\right)=(k-1)(\omega \mid \omega)$. Since $d: \chi_{n-1, k}^{0} \rightarrow \mathfrak{H}_{k-1}^{n}$ is one-to-one, our assertion follows.

As a direct application of above corollary and remark we obtain

$$
\star \chi_{p, k}^{0}= \begin{cases}d \chi_{n-1,1}^{0} & \text { if } p=k=0, \\ \varepsilon_{\nu} \mathbb{R} & \text { if } p=n-1, k=1 . \\ \varepsilon_{\nu} d \chi_{n-p-2, k}^{0} & \text { otherwise. }\end{cases}
$$

Proof of (3.19). We proceed as follows. We have that $\star \chi_{p, k}^{0} \subset \mathfrak{H}_{k}^{n-p}$, for $\star \Delta=\Delta \star$. Suppose that $k=0$. If $p=0$ then $\chi_{p, k}^{0}=\chi_{0,0}^{0}=\mathbb{R}$ and $\star \mathbb{R}$ is spanned by $d x^{1} \wedge \cdots \wedge d x^{n}$. This last form is clearly both closed and co-closed, so $\star \chi_{p, k}^{0}=$ 
$\star \chi_{0,0}^{0}=d \chi_{n-1,1}^{0}$. If $p>0$ then $\chi_{p, 0}^{0}=\{0\}$ (see (3.9)) and of course $d \chi_{n-p-2,0}^{0}=$ $\{0\}$, so $\varepsilon_{v} d \chi_{n-p-2,0}^{0}=\{0\}$.

Suppose that $k>0$. If $\omega \in \chi_{p, k}^{0}$ and $\omega \neq 0$ then $\star \omega$ is closed but not co-closed. Indeed, $d(\star \omega)= \pm \star \delta \star \star \omega= \pm \star \delta \omega=0$ and $\delta(\star \omega)= \pm \star d \star \star \omega= \pm \star d \omega \neq 0$, for in this case $d \omega \neq 0$ (see Proposition 3.2) and $\star$ is an isomorphism.

If $k=1$ then $\star \chi_{p, 1}^{0} \subset \varepsilon_{\nu} d \chi_{n-p-2,1}^{0} \oplus^{\perp} I_{\Delta} \chi_{n-p-1,0}^{0}$. But $\chi_{n-p-1,0}^{0} \neq 0$ iff $p=n-1$ but then $\chi_{p, 0}^{0}=\chi_{n-1,1}^{0}$ and $\varepsilon_{v} d \chi_{n-p-2,1}^{0}=\varepsilon_{v} d \chi_{-1,1}^{0}=\{0\}$, so $\star \chi_{p, 1}^{0} \subset I_{\Delta} \chi_{n-p-1,0}^{0}$. Since, in this case, $\operatorname{dim} \chi_{p, 1}^{0}=\operatorname{dim} \chi_{n-1,1}^{0}=\left(\begin{array}{l}n \\ n\end{array}\right)=1$ and $\operatorname{dim} I_{\Delta} \chi_{p, k}^{0}=\operatorname{dim} I_{\Delta} \chi_{0,0}^{0}=\operatorname{dim} \chi_{0,0}^{0}=1$ we have the equality $\star \chi_{p, 1}^{0}=$ $I_{\Delta} \chi_{n-p-1,0}^{0}$ If $p \neq n-1, I_{\Delta} \chi_{n-p-1,0}^{0}=\{0\}$ so $\star \chi_{p, 1}^{0} \subset \varepsilon_{v} d \chi_{n-p-2,1}^{0}$. But $\operatorname{dim} \chi_{p, 1}^{0}=\left(\begin{array}{c}n \\ p+1\end{array}\right)=\left(\begin{array}{c}n \\ n-(p+1)\end{array}\right)=\operatorname{dim} \chi_{n-p-2,1}^{0}=\operatorname{dim} \varepsilon_{v} d \chi_{n-p-2,1}^{0}$. Thus, $\star \chi_{p, 1}^{0}=\varepsilon_{\nu} d \chi_{n-p-2,1}^{0}$

Suppose now that $k>1$. Then $\star \chi_{p, k}^{0} \subset \varepsilon_{\nu} d \chi_{n-p-2, k}^{0}$. Let $\eta=\varepsilon_{\nu} d \omega \in$ $\varepsilon_{\nu} d \chi_{n-p-2, k}^{0}$ and $\eta \neq 0$. Then we see that $\star \eta$ is co-closed, for $\delta(\star \eta)=-\delta \star$ $d \varepsilon_{v} \omega= \pm \star d \star \star d \varepsilon_{v} \omega=0$. Moreover, if $p \leq n-2, \star \eta$ is not closed, for $d(\star \eta)=-d \star \varepsilon_{\nu} d \omega= \pm \star \delta\left(\varepsilon_{\nu} d \omega\right) \neq 0$ by Proposition 3.2 (iv). Thus $\star \eta \in \chi_{p, k}^{0}$ and therefore, $\star \chi_{p, k}^{0}=\varepsilon_{v} d \chi_{n-p-2, k}^{0}$ if $0 \leq p \leq n-2$ and $k>1$. On the other hand, if $p=n-1$ or $p=n$ then $\varepsilon_{v} d \chi_{n-p-2, k}^{0}=\{0\}$ and $\chi_{p, k}^{0}=\{0\}$.

Proposition 3.18. Let $\omega \in \chi_{p-1, k+1}^{0}$ and $\eta=d \omega \neq 0$. Then $\eta$ is not normal, i.e., $\eta^{\mathrm{T}} \neq 0$, except the case: $p=n, k=0$.

Proof. One can easily check that $r^{2}(d \omega)^{\mathrm{T}}=r^{2} d \omega-(p+k) \varepsilon_{\nu} \omega$. Thus, $d \omega$ is normal iff $r^{2} d \omega=(p+k) \varepsilon_{\nu} \omega$. We have,

$$
\left(r^{2} d \omega \mid r^{2} d \omega\right)=\left(r^{2} d \omega \mid(n+j) \varepsilon_{\nu} \omega\right)=2(p+k)(d \omega \mid d \omega) .
$$

On the other hand, by (3.2) we have

$$
\left(r^{2} d \omega \mid r^{2} d \omega\right)=-\left(d \omega \mid \Delta\left(r^{2} d \omega\right)\right)=2(p+2 k)(d \omega \mid d \omega) .
$$

We conclude $k=0$. Of course $p \geq 1$. Now we have

$$
0=r^{2} d \omega-p \varepsilon_{\nu} \omega=\frac{n-p}{n} r^{2} d \omega-p I_{\Delta}(p, 2) \omega .
$$

Since $d \omega \neq 0$, by Corollary 3.1, $n=p$.

\section{Dirichlet boundary problem}

\subsection{Dirichlet boundary problem for Laplace-Beltrami operator}

Observe first that (2.2) implies the following

Proposition 4.1. For each $g \in \mathcal{P}_{k}$ there exist a unique $f \in \mathcal{P}_{k}$ such that $\Delta\left(r^{2} f\right)=g$. 
Let $S$ denote the unit sphere in $\mathbb{R}^{n}$.

Proposition 4.2. If $w$ is a harmonic polynomial and $\left.w\right|_{S}=0$ then $w=0$.

Proof. We have $w=f_{k}+\ldots+f_{0}$, where $f_{j} \in \mathcal{H}_{j}, j=0, \ldots, k$, If $k=0$ our assertion is trivial. Assume that $k \geq 1$ and $f_{k} \neq 0$. Let $x \neq 0$ then $w(x /|x|)=0$, for $x /|x| \in S$. Homogeneity implies now that $0=|x|^{-k} f_{k}(x)+\ldots+|x|^{-1} f_{1}(x)+$ $|x|^{0} f_{0}(x)$. Without loss of generality we may assume that $k$ is even, so if we put $y=x$ and $y=-x$, we obtain two following equations

$$
\begin{aligned}
0= & f_{k}(y)+|y|^{2} f_{k-2}(y)+\ldots+|y|^{2 \frac{k}{2}} f_{0} \\
& +|y|\left(f_{k-1}(y)+|y|^{2} f_{k-3}(y)+\ldots+|y|^{2 \frac{k-2}{2}} f_{1}(y)\right) . \\
0= & f_{k}(y)+|y|^{2} f_{k-2}(y)+\ldots+|y|^{2 \frac{k}{2}} f_{0} \\
& -|y|\left(f_{k-1}(y)+|y|^{2} f_{k-3}(y)+\ldots+|y|^{2 \frac{k-2}{2}} f_{1}(y)\right) .
\end{aligned}
$$

They follows that $f_{k}+r^{2} f_{k-2}+\ldots+\left(r^{2}\right)^{\frac{k}{2}} f_{0}=0$, and $f_{k-1}+r^{2} f_{k-3}+\ldots+$ $\left(r^{2}\right)^{\frac{k-2}{2}} f_{1}=0$. Proposition 2.1 implies now that $f_{0}=\ldots=f_{k-2}=f_{k}=0$, and $f_{1}=\ldots=f_{k-3}=f_{k-1}=0$.

This completes the proof.

Theorem 4.3 (Dirichlet boundary problem). Let $\omega$ and $\eta$ be polynomial $p$-forms. There exists unique polynomial p-form $\varphi$ such that $\Delta \varphi=\omega$ and $\left.\varphi\right|_{S}=\left.\eta\right|_{S}$.

Theorem 4.3 is a direct consequence of 3.1 and the following:

Lemma 4.4. Let $f$ and $g$ be polynomials. The differential equation $\Delta u=f$ and $\left.u\right|_{S}=g$, has the unique polynomial solution.

Proof. Without loss of generality, we may assume that $f, g$ are homogeneous polynomials. Let, for example, $f \in \mathcal{P}_{k}$ and $g \in \mathcal{P}_{l}$. Proposition 4.1 implies, that there exists a unique polynomial $v \in \mathcal{P}_{k}$, such that $\Delta\left(r^{2} v\right)=f$. From Proposition 2.1 we have $v=v_{0}+r^{2} v_{1}+\ldots+\left(r^{2}\right)^{m_{k}} v_{m_{k}}$ and $g=g_{0}+r^{2} g_{1}+\ldots+\left(r^{2}\right)^{m_{l}} g_{m_{l}}$, where $v_{j} \in \mathcal{H}_{k-2 j}, 0 \leq j \leq m_{k}$, and $g_{j} \in \mathcal{H}_{k-2 j}, 0 \leq j \leq m_{l}$. Put $v^{\prime}=$ $v_{0}+v_{1}+\ldots+v_{m_{k}}, g^{\prime}=g_{0}+g_{1}+\ldots+g_{m_{l}}$. then $v^{\prime}, g^{\prime}$ are harmonic polynomials, $\left.v^{\prime}\right|_{S}=\left.\left(r^{2} v\right)\right|_{S}$ and $\left.g^{\prime}\right|_{S}=\left.g\right|_{S}$. Obviously, the polynomial $u=r^{2} v-v^{\prime}+g^{\prime}$ is a solution to $\Delta u=f,\left.u\right|_{S}=g$.

The uniqueness results from Proposition 4.2.

\subsection{Dirichlet problem for Laplace type operator}

Consider a Laplace type operator $L=a d \delta+b \delta d, a, b>0$. A goal of this section is the following 
Theorem 4.5 (Dirichlet boundary problem). For any $\omega, \eta \in \Lambda^{p}$, there exists the unique $\varphi \in \Lambda^{p}$ such that $L \varphi=\omega$ and $\left.\varphi\right|_{S}=\left.\eta\right|_{S}$.

Clearly, if $p=n$ or $p=0$, or $a=b$ then Theorem 4.5 reduces to the Dirichlet problem for $\Delta$. Therefore, we may suppose that $0<p<n$ and $a \neq b$.

As a consequence of 3.5 we obtain:

Proposition 4.6. For any $\omega \in \Lambda_{k}^{p}$ there exist unique $\psi \in \Lambda_{k}^{p}$ such that $L \psi=\omega$.

Lemma 4.7. Let $\omega \in \Lambda^{p}$. If $L \omega=0$ and $\left.\omega\right|_{S}=0$ then $\omega=0$.

Proof. We may write $\omega=\omega_{k}+\cdots+\omega_{0}$, where $\omega_{j} \in \mathfrak{L}_{j}^{p}$. If $k=0$ then our assertion is obvious. Let $k>0$. Suppose that $\omega_{k} \neq 0$. Corollary 3.11 implies that for any $j>0, \omega_{j}=\omega_{j}^{\prime}+I_{L}\left(\eta_{j}\right)$, where $\omega_{j}^{\prime} \in \chi_{p, j}$ and $\eta_{j} \in \chi_{p-1, j-1}$. Observe that $I_{L} \eta_{j}=I_{\Delta} \eta_{j}+c_{j} r^{2} d \eta_{j}$, where the constant $c_{j}=c_{\Delta}-c_{L}$ depends on $j$ (see (3.15)). Let

$$
\tilde{\omega}=\sum_{j=1}^{k}\left(\omega_{j}^{\prime}+I_{\Delta}\left(\eta_{j}\right)+c_{j} d \eta_{j}\right)+\omega_{0} .
$$

Clearly, $\omega$ is harmonic and $\left.\omega\right|_{S}=\left.\tilde{\omega}\right|_{S}=0$, so $\tilde{\omega}=0$. Thus $\omega_{k}^{\prime}+I_{\Delta} \eta_{k}=0$. By Corollary 3.11 it results that $\omega_{k}^{\prime}=0$ and $\eta_{k}=0$. Therefore $\omega_{k}=0$. It contradicts our assumption $\omega_{k} \neq 0$.

Proof of Theorem 4.5. We may assume that $\omega \in \Lambda_{k}^{p}$ and $\eta \in \Lambda_{l}^{p}$. Take $\psi \in \Lambda_{k+2}^{p}$ such that $L \psi=\omega$. By Corollary 3.1 there exist unique forms $\psi_{i} \in \mathfrak{H}_{k+2-2 i}^{p}$, $0 \leq i \leq m_{k+2}$ and $\eta_{j} \in \mathfrak{H}_{l-2 j}^{p}, 0 \leq j \leq m_{l}$ such that $\psi=\psi_{0}+r^{2} \psi_{1}+\cdots+$ $\left(r^{2}\right)^{m_{k+2}} \psi_{m_{k+2}}$ and $\eta=\eta_{0}+r^{2} \eta_{1}+\cdots+\left(r^{2}\right)^{m_{l}} \eta_{l}$.

Corollary 3.11 implies that for any $0 \leq i<m_{k+2}$ and $0 \leq j<m_{l}$ there exist unique $p$-forms $\psi_{i}^{\prime} \in \chi_{p, k+2-2 i}, \psi_{i}^{\prime \prime} \in \chi_{p-1, k+2-2 i-1}, \eta_{j}^{\prime} \in \chi_{p, l-2 j}$ and $\eta_{j}^{\prime \prime} \in \chi_{p-1, l-2 j-1}$ such that $\psi_{i}=\psi_{i}^{\prime}+I_{\Delta}\left(\psi_{i}^{\prime \prime}\right)$ and $\eta_{j}=\eta_{j}^{\prime}+I_{\Delta}\left(\eta_{j}^{\prime \prime}\right)$. Therefore, $\psi_{i}=\psi_{i}^{\prime}+I_{L}\left(\psi_{i}^{\prime \prime}\right)+c_{i} r^{2} d \psi_{i}^{\prime \prime}$ and $\eta_{j}=\eta_{j}^{\prime}+I_{L}\left(\eta_{j}^{\prime \prime}\right)+c_{j} r^{2} \eta_{j}^{\prime \prime}$, where $c_{j}=c_{\Delta}-c_{L}$. Hence $\left.\psi\right|_{S}=\left.\tilde{\psi}\right|_{S}$ and $\left.\eta\right|_{S}=\left.\tilde{\eta}\right|_{S}$, where

$$
\begin{aligned}
& \tilde{\psi}=\sum_{i=0}^{m_{k+2}-1}\left(\psi_{i}^{\prime}+I_{L}\left(\psi_{i}^{\prime \prime}\right)+c_{i} d \psi_{i}^{\prime \prime}\right)+\psi_{m_{k+2}}, \\
& \tilde{\eta}=\sum_{j=0}^{m_{l}-1}\left(\eta_{j}^{\prime}+I_{L}\left(\eta_{j}^{\prime \prime}\right)+c_{j} d \eta_{j}^{\prime \prime}\right)+\eta_{m_{l}} .
\end{aligned}
$$

Since $L \tilde{\psi}=L \tilde{\eta}=0$, it suffices to put $\varphi=\psi-\tilde{\psi}+\tilde{\eta}$.

The uniqueness results from Lemma 4.7.

Remark 4.8. It is worth to note that in whole section we used only the Corollary 3.11 . 


\subsection{Projection formulae}

Let $L$ be a Laplace type operator, $k \geq 0$ and $0 \leq p \leq n$. Fix $\alpha \in \mathfrak{L}_{k}^{p}$. By Theorem 3.7 there exist unique $\alpha^{1} \in \chi_{p, k}^{0}, \alpha^{2} \in d \chi_{p-1, k+1}^{0}, \alpha^{3} \in \varepsilon_{\nu} d \chi_{p-2, k}^{0}$ and $\alpha^{4} \in I_{L} \chi_{p-1, k-1}^{0}$ such that

$$
\alpha=\alpha^{1}+\alpha^{2}+\alpha^{3}+\alpha^{4} .
$$

For each $1 \leq i \leq 4$ define the projection $\pi^{i}=\pi_{L}^{i}$ by $\pi^{i}(\alpha)=\alpha^{i}$. Let $\eta^{2} \in$ $\chi_{p-1, k+1}^{0}, \eta^{3} \in \chi_{p-2, k}^{0}$ and $\eta^{4} \in \chi_{p-1, k-1}^{0}$ be such that $\alpha^{2}=d \eta^{2}, \alpha^{3}=\varepsilon_{\nu} d \eta^{3}$ and $\alpha^{4}=I_{L} \eta^{4}$. Lemma 3.4, Proposition 3.2 and Lemma 3.14 imply that if $\alpha^{i} \neq 0$ then corresponding $\eta^{i}$ is uniquely determined. If $\alpha^{i}=0$ then we put $\eta^{i}=0$. Define the maps $\sigma^{i}=\sigma_{L}^{i}, i=2,3,4$, by

$$
\sigma^{i}(\alpha)=\sigma_{L}^{i}(\alpha)=\eta^{i}
$$

Let $\mathbf{j}$ denote the identity map on $\mathfrak{L}_{k}^{p}$. Clearly, we have

$$
\pi^{1}=\mathbf{j}-\pi^{2}-\pi^{3}-\pi^{4}, \quad \pi^{2}=d \sigma^{2}, \quad \pi^{3}=\varepsilon_{v} d \sigma^{3}, \quad \pi^{4}=I_{L} \sigma^{4} .
$$

Let $\eta=\eta^{4}+d \eta^{3}$. One can easily calculate that

$$
\delta I_{L} \eta=\left(2 c_{L}(p+k-2)-(n-p+k)\right) \eta^{4}-(n-p+k) d \eta^{3},
$$

where of course, $I_{L}=I_{L}(p, k)$ and $c_{L}=c_{L}(p, k)$. Therefore,

$$
\iota_{\nu} \delta I_{L} \eta=-(n-p+k)(p+k-2) \eta^{3} .
$$

Since $\delta \alpha=\delta I_{L} \eta$ we obtain

$$
\begin{aligned}
& \sigma^{3}= \begin{cases}0, & \text { if } k=0 \text { or } p=0,1, \\
-\frac{1}{(n-p+k)(p+k-2)} \iota_{\nu} \delta, & \text { otherwise. }\end{cases} \\
& \sigma^{4}= \begin{cases}0, & \text { if } k=0 \text { or } p=0, \\
\frac{1}{2 c_{L}(p+k-2)-(n-p+k)}\left(\delta+(n-p+k) d \sigma^{3}\right), & \text { or } p=n \text { and } k=2,\end{cases}
\end{aligned}
$$

And finally,

$$
\sigma^{4}= \begin{cases}0, & \text { if } k=0 \text { or } p=0, \\ -\frac{a(p+k-2)+b(n-p+k-2)}{b(n-p+k-2)(n+2 k-2)} \delta, & \text { or } p=n \text { and } k=2, \\ -\frac{a(p+k-2)+b(n-p+k-2)}{b(n-p+k-2)(n+2 k-2)(p+k-2)} \iota_{\nu} d \delta, & \text { otherwise. }\end{cases}
$$


Let $\varphi=\alpha^{1}+d \eta^{2}$. Since $\iota_{\nu} \varphi=(p+k) \eta^{2}$ we obtain

$$
\sigma^{2}= \begin{cases}0, & \text { if } p=0, \\ \frac{1}{p+k} \iota_{v}\left(\mathbf{j}-\pi^{4}-\pi^{3}\right), & \text { otherwise. }\end{cases}
$$

In the example below we derive for given form $\alpha \in \mathfrak{H}_{3}^{1}$ corresponding $\alpha^{i}$ and $\eta^{i}$.

Example 4.9. Let $n=3$ and $\alpha$ be a (1/3)-form: $\alpha=\left(3 y z^{2}-y^{3}\right) d x+x y z d y+$ $\left(3 x^{2} y-y^{3}\right) d z$. One sees that $\alpha \in \mathfrak{H}_{3}^{1}$. Since $p=1$,

$$
\alpha^{3}=0
$$

We see that $c_{\Delta}=c_{\Delta}(1,3)=1 / 5$ and $\delta \alpha=-x z$, thus

$$
\eta^{4}=(5 / 21) x z .
$$

Hence

$$
\begin{aligned}
\alpha^{4} & =I_{\Delta} \eta^{4}=I_{\Delta}(1,3) \eta^{4} \\
& =\frac{1}{21}\left(\left(4 x^{2} z-y^{2} z-z^{3}\right) d x+5 x y z d y+\left(4 x z^{2}-x^{3}-x y^{2}\right) d z\right) .
\end{aligned}
$$

Therefore,

$$
\begin{aligned}
\eta^{2}= & \frac{1}{4} \iota_{v}\left(\alpha-\alpha^{4}\right) \\
= & \frac{1}{28}\left(-x^{3} z+21 x^{2} y z-7 x y^{3}+6 x y^{2} z+21 x y z^{2}-x z^{3}-7 y^{3} z\right) \\
\alpha^{2}= & d \eta^{2} \\
= & \frac{1}{28}\left(\left(-3 x^{2} z+42 x^{2} y z-7 y^{3}+6 y^{2} z+21 y z^{2}-z^{3}\right) d x\right. \\
& +\left(21 x^{2} z-21 x y^{2}+12 x y z+21 x z^{2}-21 y^{2} z\right) d y \\
& \left.+\left(-x^{3}+21 x^{2} y+6 x y^{2}+42 x y z-3 x z^{2}-7 y^{3}\right) d z\right)
\end{aligned}
$$

So

$$
\begin{aligned}
\alpha^{1}= & \frac{1}{12}\left(\left(-x^{2} z-18 x y z-9 y^{3}-2 y^{2} z+27 y z^{2}+z^{3}\right) d x\right. \\
& +\left(-9 x^{2} z+9 x y^{2}+4 x y z-9 x z^{2}+9 y^{2} z\right) d y \\
& \left.+\left(x^{3}+27 x^{2} y-2 x y^{2}-18 x y z-x z^{2}-9 y^{3}\right) d z\right) .
\end{aligned}
$$




\subsection{Explicit solutions}

Fix the Laplace type operator $L=a d \delta+b \delta d, a, b>0$. Let $\omega \in \Lambda^{p}$. We give a method of finding the solution to the Dirichlet Problem $L \varphi=0,\left.\varphi\right|_{S}=\left.\omega\right|_{S}$.

Suppose first that $\omega \in \mathfrak{H}_{k}^{p}$. Clearly, if $k=0,1$ we put $\varphi=\omega$. Let $k>1$ and let $\eta=\sigma^{4} \omega$. Put

$$
\omega^{\prime}=\omega+\left(1-r^{2}\right)\left(c_{L}-c_{\Delta}\right) d \eta,
$$

where, clearly, $c_{L}=c_{L}(p, k)$ and $c_{\Delta}=c_{\Delta}(p, k)$. One sees that $\left.\omega\right|_{S}=\left.\omega^{\prime}\right|_{S}$. Since (see Theorem 3.7) $L d \eta=0$ and $\omega-r^{2}\left(c_{L}-c_{\Delta}\right) d \eta=\left(\omega-I_{\Delta} \eta\right)+I_{L} \eta, L \omega^{\prime}=0$. Therefore $\varphi=\omega^{\prime}$ is the unique solution to $L \varphi=0$ and $\left.\varphi\right|_{S}=\left.\omega^{\prime}\right|_{S}$.

Fix $\omega \in \Lambda^{p}$. If $\omega=\omega_{0}+\omega_{1}+\cdots+\omega_{k}$, where $\omega_{i} \in \Lambda_{i}^{p}$ then $\varphi=\varphi_{0}+\varphi_{1}+$ $\cdots+\varphi_{l}$, where $\varphi_{i}$ is the solution to $L \varphi_{i}=0,\left.\varphi_{i}\right|_{S}=\left.\omega_{i}\right|_{S}$. So we may assume that $\omega \in \Lambda_{k}^{p}$. Suppose that $\omega$ has the following decomposition (see Corollary 3.1)

$$
\omega=\alpha_{0}+r^{2} \alpha_{1}+\cdots+\left(r^{2}\right)^{m_{k}} \alpha_{m_{k}},
$$

where $\alpha_{i} \in \mathfrak{H}_{l-2 i}^{p}$. Let $\omega_{i}^{\prime}$ be such that $L \omega_{i}^{\prime}=0$ and $\left.\omega_{i}^{\prime}\right|_{S}=\left.\alpha_{i}\right|_{S}$. Then $\varphi=\omega_{0}^{\prime}+$ $\cdots+\omega_{m_{k}}^{\prime}$ is the unique solution to the differential equation $L \varphi=0,\left.\varphi\right|_{S}=\left.\omega\right|_{S}$. So it suffices to derive $\alpha_{i}^{\prime} s$. In fact, a method of deriving of $\alpha^{i}$ reduces to an algorithm of finding spherical harmonic decomposition of homogeneous polynomial which can be found in the literature (see, for example [2, Theorem 5.21]). However, our recurrence formula seems to be more efficient for computation.

$m \geq 0$,

By (2.1) and an easy recurrence, we obtain that for any $h \in \mathcal{H}_{k}, l \geq 0$ and

$$
\Delta^{l}\left(\left(r^{2}\right)^{m} h\right)=\gamma_{k, l, m}\left(r^{2}\right)^{m-l} h
$$

where

$$
\gamma_{k, l, m}= \begin{cases}1, & \text { if } l=0 \\ (-1)^{l} 2^{l} \prod_{j=0}^{l-1}(m-j)(n+2(k+m-j-1)), & \text { if } l>0 .\end{cases}
$$

Notice that $\Delta^{l}\left(\left(r^{2}\right)^{m} h\right)=0$ if $l>m$. Put $\gamma_{k, m}=\gamma_{k, m, m}$, for simplicity.

Remark 4.10. Above formula is the very special case of the general one given in [13, Lemma 3.2 page 176]. This formula asserts how to compute $\Delta^{l}(\phi h)$ for any $h \in \mathcal{H}_{k}$ and any (smooth) radial function $\phi$.

$$
\text { Put } \omega_{i}=\alpha_{0}+r^{2} \alpha_{1}+\cdots+\left(r^{2}\right)^{i} \alpha_{i}, 0 \leq i \leq m_{k} \text {. Clearly, } \omega_{m_{k}}=\omega, \omega_{0}=\alpha_{0}
$$
and for any $1 \leq i \leq m_{k}, \omega_{i-1}=\omega_{i}-\left(r^{2}\right)^{i} \alpha_{i}$. By (3.2) and the definition of $\gamma_{k, l, m}$ we obtain

$$
\alpha_{i}=\frac{1}{\gamma_{k-2 i, i}} \Delta^{i} \omega_{i}, \quad \text { for } i=0, \ldots, m_{k},
$$

where of course $\Delta^{s}=\Delta \circ \cdots \circ \Delta(s$ times $)$. 
Example 4.11. Let $n=3$. Consider polynomial $f, f(x, y, z)=4 y z^{2}+x^{2} y$. Clearly $f \in \mathcal{P}_{3}$ and $\Delta f(x, y, z)=-10 y$. Let $f=h_{0}+r^{2} h_{1}, h_{i} \in \mathcal{H}_{3-2 i}, i=0,1$. One sees that $m_{3}=[3 / 2]=1$ and $\gamma_{3-2 \cdot 1,1}=-10$. Therefore, $h_{1}(x, y, z)=$ $(-10 y) /(-10)=y$. Hence

$$
f(x, y, z)=3 y z^{2}-y^{3}+\left(x^{2}+y^{2}+z^{2}\right) y .
$$

Example 4.12. Let $n=3$ and $\omega=\left(4 y z^{2}+x^{2} y\right) d x+x y z d y+\left(3 x^{2} y-y^{3}\right) d z$. Solve the Dirichlet problem

$$
S^{\star} S \varphi=0 \text { and }\left.\varphi\right|_{S}=\left.\omega\right|_{S} .
$$

Since $n=3$ (see Section 3.1), $S^{\star} S=(2 / 3) d \delta+(1 / 2) \delta d$. We see ( $c f$. Example 4.9 and 4.11) that $\omega=\alpha+r^{2} y d x$, where $\alpha$ was form from Example 4.9.

Since $k=3$ and $p=1$ (see (3.15)) $c_{S^{\star} S}=11 / 34$ and $c_{\Delta}=1 / 5$. Since $\eta^{4}=(5 / 21) x z$ (cf. Example 4.9) the unique solution to (4.3) is $\varphi=\alpha+\left(c_{S^{\star} S}-\right.$ $\left.c_{\Delta}\right)\left(1-r^{2}\right) d \eta^{4}+y d x$, i.e.,

$$
\begin{aligned}
\varphi= & \frac{1}{34}\left(-x^{2} z-34 y^{3}-y^{2} z+102 y z^{2}+34 y-z^{3}+z\right) d x+x y z d y \\
& +\frac{1}{34}\left(-x^{3}+102 x^{2} y-x y^{2}-x z^{2}+x-34 y^{3}\right) d z .
\end{aligned}
$$

\section{Our decompositions from the rotation group point of view}

In the whole section we assume that $n \geq 3$. Because of representation theory it is convenient to consider complex vector spaces, so we pass to complexifications. More precisely, we complexify all spaces, maps, and extend $(\cdot, \cdot)_{p, k}$ to the Hermitian inner product. For example, since now, $\Lambda_{k}^{p}$ will denote the complexification of the space of $(p / k)$-forms in $\mathbb{R}^{n}$. Moreover we set, $\bigwedge^{p}=\left(\bigwedge^{p}\left(\mathbb{R}^{n}\right)^{\star}\right) \otimes_{\mathbb{R}} \mathbb{C}$. In fact, we have $\bigwedge^{p}=\Lambda_{0}^{p}$.

\subsection{Preliminaries}

Consider the rotation group $G=G_{n}=\mathrm{SO}(n)$, and let $s=[n / 2]$. Recall that if $\omega$ is any $p$-form on $\mathbb{R}^{n}$ or on $S$ then the natural action of $G$ on $\omega$ is given by $g \omega=\left(g^{-1}\right)^{\star} \omega$. Under this action all considered operators $d, \delta, \iota_{v}, \varepsilon_{v}$ and $\star$ are $G$-map, i.e., they commute with the $G$-action.

If $m=\left(m_{1}, \ldots, m_{s}\right)$, is a dominant weight of $G$, i.e., $m_{j}$ 's are integers that satisfy $m_{1} \geq \cdots m_{s} \geq 0$ if $n=2 s+1$ and $m_{1} \geq \cdots m_{s-1} \geq\left|m_{s}\right|$ if $n=2 s$, then $R_{n}(m)$ will denote the irreducible representation of $G$ having highest weight $m$. We will denote by $1_{j}$ (respectively $0_{j}$ ) the sequence of $j 1$ 's (respectively 0 's).

If $R$ is a representation on $V$ we will sometimes identify $R$ and $V$, writing $R=V$. If $V=R_{n}(m)$ then the space of all highest weight vector (together with zero vector), which is necessarily one-dimensional, will be denoted by hwv $(V)$. 
The following examples are classical:

(1) Let $V=\mathcal{H}_{k}$ then $V$ is irreducible, $V=R_{n}\left(k, 0_{s-1}\right)$ and $\operatorname{hwv}\left(\mathcal{H}_{k}\right)=\operatorname{span}\left(\left(x^{1}+\right.\right.$ $\left.\left.i x^{2}\right)^{k}\right)$.

(2) Let $V=\bigwedge^{p}$. If $p<n / 2$ (respectively $\left.p>n / 2\right)$ then $V=R_{n}\left(1_{p}, 0_{s-p}\right)$ and $\operatorname{hwv}(V)=\operatorname{span} \zeta^{p}$ where $\zeta^{p}=\left(d x^{1}+i d x^{2}\right) \wedge \cdots \wedge\left(d x^{2 p-1}+i d x^{2 p}\right)$ (respectively $\operatorname{hwv}(V)=\operatorname{span}\left(\star \zeta^{n-p}\right)$ ). If $p=n / 2$ then $V$ is reducible, $V=$ $V_{+} \oplus V_{-}$with $V_{+}=R_{n}\left(1_{s}\right)$ and $V_{-}=R_{n}\left(1_{s-1},-1\right), \operatorname{hwv}\left(V_{+}\right)=\operatorname{span} \zeta^{s}$ and $\operatorname{hwv}\left(V_{-}\right)=\operatorname{span} \zeta_{-}^{s}$ where $\zeta_{-}^{s}=\zeta^{s-1} \wedge\left(d x^{n-1}-i d x^{n}\right)$.

(3) Let $V=\mathfrak{H}_{k}^{p}$ and $p>0$. Then $V$ is isomorphic, as a representation, to the tensor product $\mathcal{H}_{k} \otimes \bigwedge^{p}$. Therefore, $V$ is reducible in general. We distinguish the irreducible component $V^{\prime}$ of $V$ generated by the form $\varphi^{p, k}=\left(x^{1}+i x^{2}\right)^{k} \zeta^{p}$ if $p \leq s$ and $\left(x^{1}+i x^{2}\right)^{k} \star \zeta^{n-p}$ if $p>s$. Then $V^{\prime}=R_{n}\left(k+1,1_{p-1}, 0_{s-p}\right)$ if $p \leq s, V^{\prime}=R_{n}\left(k+1,1_{n-p-1}, 0_{s-n+p}\right)$ if $s<p \leq n-1$ and $V^{\prime}=R_{n}\left(k, 0_{s-1}\right)$ if $p=n$. Moreover, $\varphi^{p, k}$ is a highest weight vector of $V^{\prime}$. If $n=2 s$ and $p=s$ we also distinguish a subspace $V_{-}^{\prime}$. This is the smallest irreducible subspaces of $V$ containing $\varphi_{-}^{s, k}=\left(x^{1}+i x^{2}\right)^{k} \zeta_{-}^{s-1}$. We have, $V_{-}^{\prime}=R_{n}\left(k+1,1_{s-2},-1\right)$ and $\varphi_{-}^{s, k}$ is a highest weight vector of $V_{-}^{\prime}$.

Denote by $\pi_{p, n}$ the natural action of $G$ on the Hilbert space $\Gamma^{p}=\Gamma\left(E^{(p)}\right)$ of all square integrable sections of the bundle $E^{(p)}=\bigwedge^{p} T^{\star} S$, which are, in fact, square integrable $p$-forms on $S .\left(E^{(0)}\right.$ is the trivial bundle $S \times \mathbb{R}$ and $\Gamma\left(E^{(0)}\right)$ is equal to $L^{2}(S)$ ). Points (a)-(e) below describe all irreducible subrepresentations of $\pi_{p, n}$. A proof based on the Frobenius reciprocity theorem and branching theorem may be found in [4, Theorem A, page 137].

The irreducible representations of $G$ occurring in $\pi_{p, n}$ are as follows:

(a) $R_{n}\left(k, 0_{s-1}\right), k \geq 0$, if $p=0$ or $p=n-1$.

(b) $R_{n}\left(k, 1_{p-1}, 0_{s-p}\right)$ and $R_{n}\left(k, 1_{p}, 0_{s-p-1}\right), k \geq 1$, if $1 \leq p<s-1$ or if $n=2 s+1$ and $p=s-1$.

(c) $R_{n}\left(k, 1_{n-p-1}, 0_{s+p-n}\right)$ and $R_{n}\left(k, 1_{n-p-2}, 0_{s+p+1-n}\right), k \geq 1$ if $s<p<n-1$.

(d) $R_{n}\left(k, 1_{s-1}\right), R_{n}\left(k, 1_{s-2}, 0\right)$ and $R_{n}\left(k, 1_{s-2},-1\right), k \geq 1$, if $n=2 s$ and $p=s-1$ or $p=s$.

(e) $R_{n}\left(k, 1_{s-1}\right), k \geq 1$, if $n=2 s+1$ and $p=s$.

The irreducible representations from (a)-(d) occur with multiplicity one. The representations from (e) occur with multiplicity two.

\subsection{Irreducible decomposition of $\mathfrak{L}_{k}^{p}$}

As we have seen, in Section 3.3 if $L=a d \delta+b \delta d, a, b>0$ is a Laplace type operator then $\mathfrak{L}_{k}^{p}=\operatorname{ker} L \cap \Lambda_{k}^{p}$ splits as (see (3.16))

$$
\mathfrak{L}_{k}^{p}=\chi_{p, k}^{0} \oplus^{\perp} d \chi_{p-1, k+1}^{0} \oplus^{\perp} \varepsilon_{\nu} d \chi_{p-2, k}^{0} \oplus^{\perp} I_{L}(p, k) \chi_{p-1, k-1}^{0} .
$$


Since $\Lambda_{k}^{p}$ is $G$-invariant subspace, $\chi_{p, k}^{0}=\operatorname{ker} \delta \cap \operatorname{ker} \iota_{v} \cap \Lambda_{k}^{p}$ is. Therefore, each component in the above decomposition is $G$-invariant subspace. Our aim is to check when (3.16) is irreducible. In the light of Proposition 3.2 and Corollary 3.11 we know when $G$-maps $d, \varepsilon_{v} d$, and $I_{L}$ are one-to-one. Thus, only we have to do is to show when $\chi_{p, k}^{0}$ is irreducible. By (3.19), Proposition 3.2 and $G$-equivariantness of $d, \varepsilon_{v}$ and $\star$ we obtain:

Proposition 5.1. Representation $\chi_{p, k}^{0}$ is equivalent to $\chi_{n-1,1}^{0}$ if $p=k=0$ and to $\chi_{n-p-2, k}^{0}$ if neither $p=k=0$ nor $p=n-1, k=1$.

Suppose $0 \leq p \leq n-1$. If $\chi_{p, k}^{0} \neq \mathbb{R}$, i.e., $p>0$ or $k>0$ then representations $\chi_{p, k}^{0}$ and $d \chi_{p, k}^{0}$ are equivalent.

Let $i_{S}$ denote the inclusion map $S \rightarrow \mathbb{R}^{n}$. The pull-back $i_{S}^{\star}$ is a $G$-map. Similarly, tangential projection $\omega \mapsto \omega^{T}$ and restriction $\left.\omega \mapsto \omega\right|_{S}$ are $G$-maps, so the map $\left.\omega \mapsto \omega^{T}\right|_{S}$ is. Clearly, we may identify, $\left.\omega^{T}\right|_{S}$ and $i_{S}^{\star} \omega$. Suppose that $\omega$ is a homogeneous polynomial form. If $\omega$ is not normal, i.e., $\omega^{T} \neq 0$, then by homogeneity $\left.\omega^{T}\right|_{S} \neq 0$. If, in addition, $\omega$ is tangential (for example if $\omega \in \chi_{p, k}^{0}$ ) then we may identify $\omega$ with $\left.\omega^{T}\right|_{S}$ or equivalently with $i_{S}^{\star} \omega$ :

$$
i_{S}^{\star} \chi_{p, k}^{0}=\chi_{p, k}^{0} \text {. }
$$

If $\eta=d \omega, \omega \in \chi_{p-1, k+1}^{0}, p<n$ and $\eta \neq 0$ then by Proposition $3.18 \eta^{T} \neq 0$, and (outside 0$) \eta^{T}=(d \omega)^{T}=d \omega-\left(1 / r^{2}\right)(p+k) \varepsilon_{\nu} \omega$. So, $\left.\eta^{T}\right|_{S}=(d \omega-(p+$ k) $\left.\varepsilon_{\nu} \omega\right)\left.\right|_{S}$. Consequently, under identification $i_{S}^{\star} \eta=\left.\eta^{T}\right|_{S}$ we have

$$
i_{S}^{\star}\left(d \chi_{p-1, k+1}^{0}\right)=\left\{\left.\left(d \omega-(p+k) \varepsilon_{v} \omega\right)\right|_{S}: \omega \in \chi_{p-1, k+1}^{0}\right\} .
$$

We have

$$
i_{S}^{\star} \chi_{p, k}^{0} \cap i_{S}^{\star}\left(d \chi_{q-1, l+1}^{0}\right)=\{0\} .
$$

Proof of (5.1). We may assume that $0 \leq p, q \leq n$. If $p \neq q$ then our assertion is obvious. Suppose that $p=q$. If $p=0, i_{S}^{\star}\left(d \chi_{p-1, l+1}^{0}\right)=\{0\}$. Take $p>0$. There exist $\alpha \in \chi_{p, k}^{0}$ and $\omega \in \chi_{p-1, l+1}^{0}$ such that $\alpha=d \omega-(p+l) \varepsilon_{\nu} \omega$ on $S$. But $d \omega-(p+l) \varepsilon_{\nu} \omega=\left(1+c_{\Delta}(p, l+2) r^{2}\right) d \omega+I_{\Delta}(p, l+2) \omega$. Thus on $S$,

$$
\alpha=\left(1+c_{\Delta}(p, l+2)\right) d \omega+I_{\Delta}(p, l+2) \omega .
$$

Since all forms from (5.2) are harmonic, equality (5.2) holds on the whole $\mathbb{R}^{n}$. Now by (3.17) it follows that $\alpha=\omega=0$.

Let $\varphi^{p . k}$ and $\varphi_{-}^{s, k}$ be as in example (3). One can verify that if neither $p=0$, $k>0$ nor $p=n, k>0$ this forms are both closed and co-closed. Thus by Corollary 3.16 it follows that if $k \geq 0,0<p<n$ or $k=0, p=n$,

$$
\varphi^{p . k} \in d \chi_{p-1, k+1}^{0},
$$

and $\varphi^{0,0} \in \mathbb{R}$ if $p=0$. 
Define now forms $\psi^{p-1, k+1}$ and $\psi_{-}^{s-1, k+1}$ as follows; $\psi^{p-1, k+1}=\iota_{\nu} \varphi^{p, k}$ and $\psi_{-}^{s-1, k+1}=\iota_{\nu} \varphi_{-}^{s, k}$. Moreover, we put $\psi^{p, 0}=0,(p>0)$ and $\psi^{0,0}=1$. By definitions follows that $\psi^{0, k}=\varphi^{0, k}$. Moreover we see that

$$
\psi^{p-1, k+1} \in \chi_{p-1, k+1}^{0}
$$

except the case $p=n$ and $k>0$. Similarly,

$$
\psi_{-}^{s-1, k+1} \in \chi_{s-1, k+1}^{0} \text {. }
$$

We conclude the following:

Proposition 5.2. Let $0 \leq p \leq n$ and $k \geq 0$. Then we have $\chi_{p, k}^{0}=\{0\}$ iff either $p>0, k=0$ or $p=n$ or $p=n-1, k \neq 1$. Except this cases $\chi_{p, k}^{0} \neq\{0\}$, for $\psi^{p, k} \in \chi_{p, k}^{0}$ and $\psi^{p, k} \neq 0$.

By an easy calculation we obtain:

Proposition 5.3. For any $k \geq 0$ we have

$$
\left(\varphi^{s, k} \mid \varphi_{-}^{s, k}\right)=0 \quad \text { and } \quad\left(\psi^{s-1, k} \mid \psi_{-}^{s-1, k}\right)=0 .
$$

Let $U_{p, k}$ and $W_{p, k}$ be the smallest $G$-irreducible subspaces of $\Gamma^{p}$ containing $\left.\left(\varphi^{p, k}\right)^{T}\right|_{S}$ and $\psi^{p, k}$, respectively. Clearly, $U_{n, k}=W_{n, k}=\{0\}$ and $U_{0, k}=W_{0, k}$. We also define $U_{s, k}^{-}$and $W_{s-1, k}^{-}$as the smallest $G$-irreducible subspaces of containing $\left.\left(\varphi_{-}^{s, k}\right)^{T}\right|_{S}$ and $\psi_{-}^{s-1, k}$, respectively. From the example (3) and considerations above

$$
\begin{aligned}
& U_{p, k}=\left\{\begin{array}{llr}
R_{n}\left(k, 0_{s-1}\right) & \text { if } r & p=0, k \geq 0, \\
R_{n}\left(k+1,1_{p-1}, 0_{s-p}\right) & \text { if } 0<p \leq s, k \geq 0, \\
R_{n}\left(k+1,1_{n-p-1}, 0_{s+p-n}\right) & \text { if } s<p \leq n-1, k \geq 0 .
\end{array}\right.
\end{aligned}
$$

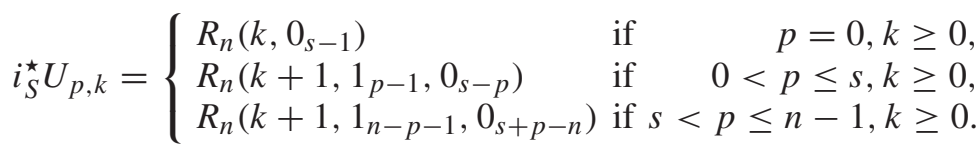

$$
\begin{aligned}
& W_{p, k}= \begin{cases}R_{n}\left(k, 0_{s-1}\right) & \text { if } \quad p=0, k \geq 0, \\
R_{n}\left(0_{s}\right) & \text { if } p=n-1, k=1, \\
R_{n}\left(k, 1_{p}, 0_{s-p-1}\right) & \text { if } 0<p \leq s-1, k \geq 1, \\
R_{n}\left(k, 1_{n-p-2}, 0_{s+p-n+1}\right) & \text { if } s \leq p<n-1, k \geq 1 .\end{cases}
\end{aligned}
$$

We also have

$$
\begin{aligned}
U_{s, k}^{-} & =R_{n}\left(k+1,1_{s-2},-1\right), & & k \geq 0, n=2 s, \\
i_{S}^{\star} U_{s, k}^{-} & =R_{n}\left(k+1,1_{s-2},-1\right), & & k \geq 0, n=2 s, \\
W_{s-1, k}^{-} & =R_{n}\left(k, 1_{s-2},-1\right), & & k \geq 1, n=2 s .
\end{aligned}
$$


Taking into account (5.1) and comparing representations $U_{p, k}, W_{p, k}, U_{s, k}^{-}$and $W_{s-1, k}^{-}$with representations occurring in (a)-(e) above we conclude:

Theorem 5.4. Suppose that $0 \leq p \leq n-1$ and $k \geq 0$. The subreperesntation $\chi_{p, k}^{0}$ of $\pi_{p, n}$ is reducible iff $n=2 s, p=s-1, k \geq 1$. In this case $\chi_{s-1, k}^{0}$ splits as a sum of two irreducible representations. More precisely, we have

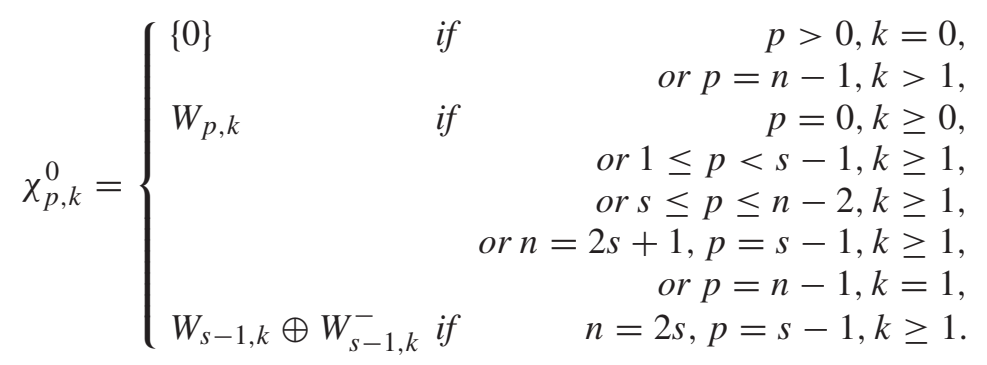

Now we are able to give a full characterization of $\mathfrak{L}_{k}^{p}$ from the representation theory point of view.

Theorem 5.5. $\mathfrak{L}_{k}^{p}$ has the following decomposition onto direct sum of nonempty $G$-irreducible subspaces;

If $p=0, k \geq 0$ then

$$
\mathfrak{L}_{k}^{0}=W_{0, k}
$$

If $p \geq 1$ and $n=2 s+1$ then

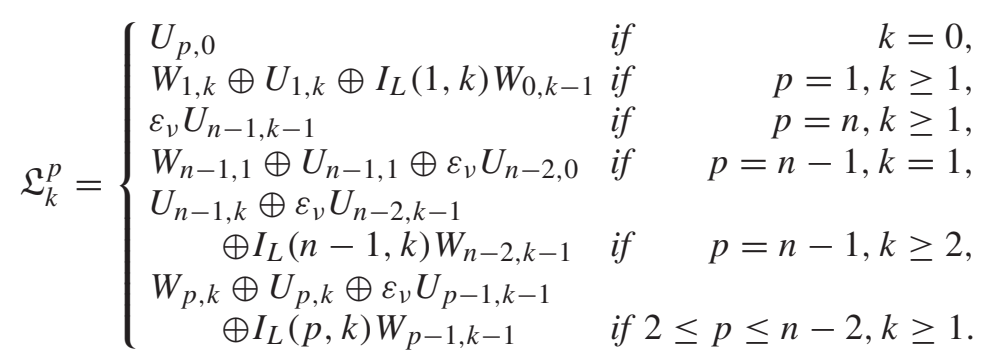

If $n=2 s$ then $s \geq 2(n \geq 3)$ and the decomposition of $\mathfrak{L}_{k}^{p}$ is the same as above except the cases $p=s-1, p=s$ and $p=s+1$ where we have

$$
\begin{gathered}
\mathfrak{L}_{k}^{s-1}= \begin{cases}W_{1, k} \oplus W_{1, k}^{-} \oplus U_{1, k} \oplus I_{L}(1, k) W_{0, k-1} & \text { if } s=2, k \geq 1 \\
W_{s-1,1} \oplus W_{s-1,1}^{-} \oplus U_{s-1,1} \oplus \varepsilon_{\nu} U_{s-2,0} & \text { if } s \geq 3, k=1, \\
W_{s-1, k} \oplus W_{s-1, k}^{-} \oplus U_{s-1, k} & \\
\quad \oplus \varepsilon_{v} U_{s-2, k-1} \oplus I_{L}(s-1, k) W_{s-2, k-1} & \text { if } s \geq 3, k \geq 2,\end{cases} \\
\mathfrak{L}_{k}^{s}=\left\{\begin{array}{cc}
U_{s, 0} \oplus U_{s, 0}^{-} & \text {if } k=0, \\
W_{s, 1} \oplus U_{s, 1} \oplus U_{s, 1}^{-} \oplus \varepsilon_{\nu} U_{s-1,0} & \text { if } k=1, \\
W_{s, k} \oplus U_{s, k} \oplus U_{s, k}^{-} \oplus \varepsilon_{\nu} U_{s-1, k-1} & \text { if } k \geq 2,
\end{array}\right.
\end{gathered}
$$




$$
\mathfrak{L}_{k}^{s+1}= \begin{cases}U_{s+1,0} & \text { if } k=0, \\ W_{s+1,1} \oplus U_{s+1,1} \oplus \varepsilon_{v} U_{s, 0} \oplus \varepsilon_{v} U_{s, 0}^{-} & \text {if } k=1, \\ W_{s+1, k} \oplus U_{s+1, k} \oplus \varepsilon_{v} U_{s, k-1} \oplus \varepsilon_{v} U_{s, k-1}^{-} & \\ \quad \oplus I_{L}(s+1, k) W_{s, k-1} & \text { if } k \geq 2 .\end{cases}
$$

Proof. It suffices to apply Theorem 5.4, Proposition 5.2, 3.2, 5.3 and Lemma 3.10 to the decomposition (3.16).

\section{References}

[1] L. V. Ahlfors, Quasiconformal deformations and mappings in $\mathbb{R}^{n}$, J. Anal. Math. 30 (1976), 74-97.

[2] S. AXler, P. Bourdon and W. Ramey, "Harmonic Function Theory", Springer-Verlag, New York, 2001.

[3] R. R. COIFMAN and G. WeISS, Representations of compact groups and spherical harmonics, Enseign. Math. 14 (1969), 121-175.

[4] G. B. Folland Harmonic analysis of the de Rham complex on the sphere, J. Reine Angew. Math. 398 (1989), 130-143.

[5] I. KolÁř , P. W. Michor and J. Slovák, "Natural Operations in Differential Geometry", Springer-Verlag, Berlin-Heidelberg, 1993.

[6] A. KoRÁNYI and S. VÁGI, Group theoretic remarks on Riesz system on balls, Proc. Amer. Math. Soc. 85 (1982), 200-205.

[7] N. V. KRYLOV, "Lectures on Elliptic and Parabolic Equations in Hölder Spaces", Graduate Studies in Mathematics, Vol. 12, American Mathematical Society, Providence, RI, 1996.

[8] A. LiPows KI, Boundary problems for the Ahlfors operator, (in Polish), Ph.D. Thesis, Lódź University, (1996), 1-55.

[9] A. PIerZChAlski, "Geometry of Quasiconformal Deformations of Riemannian Manifolds", Łódź University Press, 1997.

[10] A. Pierzchalski, Ricci curvature and quasiconformal deformations of a Riemannian manifold, Manuscripta Math. 66 (1989), 113-127.

[11] H. M. ReImann, Rotation invariant differential equation for vector fields, Ann. Scuola Norm. Sup. Pisa Cl. Sci. 9, (1982), 160-174.

[12] E. M. STEIN and G. WeIss, "Fourier Analysis on Euclidean Spaces", Princeton University Press, 1971.

[13] A. Strasburger, Differential operators of gradient type associated with spherical harmonics, Ann. Polon. Math. 53 (1991), 161-183.

[14] H. WEYL, Eigenschwingungen eines beliebig gestatleten elastischen Korpers, Rend. Circ. Mat. Palermo 39 (1915), 1-50.

[15] K. YANO, "Integral Formulas in Riemannian Geometry", Marcel Dekker INC, New York, 1970.

Faculty of Mathematics

Łódź University

Banacha 22

90-238 Lódź (Poland)

wojciech@math.uni.lodz.pl 